\title{
Millicharged Dark Matter Detection with Ion Traps
}

\author{
Dmitry Budker®, ${ }^{1,2,3}$ Peter W. Graham $\odot,{ }^{4,5}$ Harikrishnan Ramani $\odot,{ }^{4, *}$ Ferdinand Schmidt-Kaler $\odot,{ }^{1,2}$ \\ Christian Smorra, ${ }^{6}$ and Stefan Ulmer ${ }^{7}{ }^{7}$ \\ ${ }^{1}$ Johannes Gutenberg-Universität Mainz, 55128 Mainz, Germany \\ ${ }^{2}$ Helmholtz-Institut, GSI Helmholtzzentrum für Schwerionenforschung, Mainz 55128, Germany \\ ${ }^{3}$ Department of Physics, University of California, Berkeley, California 94720, USA \\ ${ }^{4}$ Stanford Institute for Theoretical Physics, Stanford University, Stanford, California 94305, USA \\ ${ }^{5}$ Kavli Institute for Particle Astrophysics \& Cosmology, Stanford University, Stanford, California 94305, USA \\ ${ }^{6}$ Institute of Physics, Johannes Gutenberg University Mainz, Mainz, Germany \\ ${ }^{7}$ RIKEN, Fundamental Symmetries Laboratory, Wako, Saitama 351-0198, Japan
}

(Received 27 August 2021; accepted 1 February 2022; published 24 February 2022)

\begin{abstract}
We propose the use of trapped ions for the detection of millicharged dark matter. Millicharged particles will scatter off the ions, giving a signal either in individual events or in the overall heating rate of the ions. Ion traps have several properties that make them ideal detectors for such a signal. First, ion traps have demonstrated significant isolation of the ions from the environment, greatly reducing the background heating and event rates. Second, ion traps can have low thresholds for the detection of energy deposition, down to approximately the nanoelectronvolt range. Third, since the ions are charged, they naturally have large cross sections for scattering with the millicharged particles, this being further enhanced by the low velocities of the thermalized millicharged particles. Despite ion-trap setups being optimized for other goals, we find that existing measurements put new constraints on millicharged dark matter that are many orders of magnitude beyond previous bounds. For example, for a millicharged dark matter mass $m_{Q}=$ $10 \mathrm{GeV}$ and charge $10^{-3}$ of the electron charge, ion traps limit the local density to be $n_{Q} \lesssim 1 \mathrm{~cm}^{-3}$, a factor of approximately $10^{8}$ better than current constraints. Future dedicated ion-trap experiments could reach even further into unexplored parameter space.
\end{abstract}

DOI: 10.1103/PRXQuantum.3.010330

\section{INTRODUCTION}

Particles that carry a fraction of the electron charge, $Q=$ $\epsilon e$, also called millicharged particles (mCPs), are an elegant extension to the standard model (SM). The discovery of their simplest iteration, without the presence of a dark photon, would be a violation of the charge-quantization hypothesis. Alternatively, mCPs could also arise as charges quantized under a dark force that mixes kinematically with the SM photon [1].

Millicharged particles have attracted significant interest and there have been many attempts to detect them. Collider and beam-dump experiments have been performed to search for mCPs and null results have led to strong

\footnotetext{
*hramani@stanford.edu
}

Published by the American Physical Society under the terms of the Creative Commons Attribution 4.0 International license. Further distribution of this work must maintain attribution to the author(s) and the published article's title, journal citation, and DOI. limits on their parameter space in the megaelectronvolt to teraelectronvolt mass range. These include the Large Electron-Positron Collider (LEP) [2], the SLAC millicharge experiment [3], neutrino experiments [4], the ArgoNeuT experiment [5], the MilliQan pathfinder experiment at the Large Hadron Collider (LHC) [6], and the Big European Bubble Chamber (BEBC) beam-dump experiment [7]. A plethora of experiments have been proposed to improve these tests and search for still smaller charges [8-11]. At lower masses below a megaelectronvolt, stringent limits arise due to the absence of anomalous emission in stellar environments $[12,13]$. The prospect of mCPs making up some or all of the dark matter (DM) has also received a lot of interest over the years. There are robust predictions for their relic density from the early universe $[14,15]$, as well as numerous ways to detect them [16-27] depending on their mass $m_{Q}$ and their charge. Subcomponent-millicharge DM (mCDM) has also been invoked to explain several recent experimental anomalies [28-33].

Since mCPs interact with the SM particles through a massless mediator, their momentum transfer cross section 
can be large at small velocities. As a result, $\mathrm{mCDM}$ with charge large enough to scatter in the atmosphere or the rock along its path to the direct-detection experiments, rapidly loses its kinetic energy and thermalizes with the environment. When it eventually reaches a direct-detection experiment, it does not possess enough energy to deposit in the detector and it cannot be observed in such experiments [34]. However, these mCPs, which are now cooled to the ambient temperature, get trapped due to Earth's gravity and build up for the duration of the Earth's existence. This can lead to mCP densities on Earth up to 14 orders of magnitude larger than that of the virial population in the galaxy [35]. This slow, albeit dense, population requires novel detection strategies, some of which include mCP particle-antiparticle annihilation in a large-volume detector [35] and the acceleration of mCPs present in electrostatic accelerator tubes to higher energies, sufficient for subsequent direct detection [35]. If the charge is large enough, negatively charged $\mathrm{mCPs}$ can bind with large positively charged SM nuclei, thereby creating fractional charge for a macroscopic material. Searches for such fractional charges bound to a sample material include Millikan-like oil-drop experiments [36], as well as more recent levitation experiments with microspheres [37,38]. However, these limits hinge critically on the assumption that the negatively charged mCPs bind to matter, thereby restricting their validity to only large charges and masses fulfilling the relation $\epsilon \gtrsim \sqrt{2 T / Z^{2} \alpha^{2} \mu}[35,38]$. Here, $T$ is the ambient temperature, $\mu$ is the reduced mass of the DM SM nuclear system, and $Z$ is the atomic number of the nucleus.

In this work, we point out an alternative search strategy; the remarkably stable trapped ions developed for metrology and quantum information science are ideal targets for the detection of an ambient thermalized $\mathrm{mCP}$ population. There is a long history of using trapped charged SM particles for particle-physics applications. Trapped ions have been used to measure the anomalous magnetic moment of the electron [39] and the electric dipole moment of the electron [40], as well as proton and antiproton magnetic moments [41,42]. Ion traps have also been used to constrain the time variation of fundamental constants, which can be induced by ultralight bosonic dark matter $[43,44]$, and ions in traps are also among the most promising candidates for qubits in order to realize quantum computing [45].

For all of the applications listed above and especially for magnetic moment measurements and quantum computing, it is important to keep the ion trapped for a sufficiently long duration without heating from the surroundings. In the past couple of decades, there has been remarkable progress in reduction of the measured heating rate of trapped ions [46]. Further progress is expected and is an active area of research in order to achieve scalability of multi-ion systems and an increased sampling rate in precision measurements. As explained below, these properties make ion traps an ideal candidate for detecting mCPs.

\section{A. Summary of findings}

We propose the use of ion traps as detectors for $\mathrm{mCP}$ dark matter. While an individual ion constitutes a much smaller target mass than any other dark matter directdetection experiment, a trapped ion has significant advantages for the detection of mCPs, including isolation from the environment, a lower energy threshold for detection, and a larger scattering cross section with mCPs. These advantages outweigh the small target mass, allowing ion traps to reach many orders of magnitude beyond other detection methods for mCPs.

Significant effort has been put into isolating trapped ions from their environments. Thus trapped ions are now sensitive to small energy depositions down to approximately the nanoelectronvolt range. The dense thermal-gas mCPs, if they exist on Earth, would permeate the detector and can scatter off the ion.

The mCPs are thermalized with the walls of the detector, which is held at a temperature much higher than the temperature of the trapped ion. Thus, the higher-energy mCPs can transfer kinetic energy that can be detected either as a single jump of the trapped ion or an accumulation of several scatter events, resulting in heating of the ion. We discuss both types of signals. The high degree of isolation of the ion achievable in traps makes them sensitive to scattering rates for $\mathrm{mCDM}$ over a wide range of parameter space.

Ion traps also make excellent mCDM detectors because of their low energy thresholds, which are set by the energylevel spacings in the trap that can be as low as approximately the nanoelectronvolt range. This allows detection of single scattering events with this energy and it also allows such low-energy scatters to contribute to the heating rate.

Furthermore, because they are charged, ions make excellent targets for $\mathrm{mCP}$ scattering. mCPs scatter with ions via Rutherford scattering, which is greatly enhanced at small relative velocities and momentum transfers. Since the mCPs are thermalized with the walls, they generally have much lower velocities than virialized dark matter. The corresponding boost to the scattering cross section combined with the large number density of the thermalized mCPs makes direct detection with single ions viable.

The rest of this paper is organized as follows. In Sec. II, we provide a description of ion traps. In Sec. III, we give an overview of the $\mathrm{mCP}$ density on Earth, as well as their dynamics, including propagation through the trap. The detection signals are explained in Sec. IV and results and projections are presented in Sec. V. The differences 
compared to an alternative proposal for detecting mCPs [47] are also found in this section. We conclude with a discussion in Sec. VI.

\section{ION TRAPS}

Traps for (single) charged particles belong to the basic toolkit of atomic, molecular, and optical physics [48]. They have wide applications in the determination of atomic masses and fundamental constants, in precision measurements to test fundamental symmetries, and in quantum information technology. Typical ingredients common to all trap experiments are shown in Fig. 1. The core of the experiments is usually comprised of sets of electrodes supplied by ac and dc voltages, optionally placed in strong magnetic fields. The electrodes are mounted in vacuum chambers. In some cryogenic trap experiments, pressures on the level of $<10^{-18}$ mbar are achieved, which provides ultralong particle-storage times [49] and enables nondestructive long-term studies at low background. Thermal shielding and an additional insulation vacuum chamber usually surround the inner vacuum chamber. The trapped particles are manipulated, cooled, and excited via laser, microwave, and radio-frequency (rf) drives. The experimental signals for ultrasensitive precision studies, frequency measurements, and the monitoring of quantum information-processing protocols are either image currents picked up by sensitive detection circuits or fluorescence signals. The signals are acquired and processed with spectrum analyzers and charge-coupled-device (CCD) cameras, respectively.

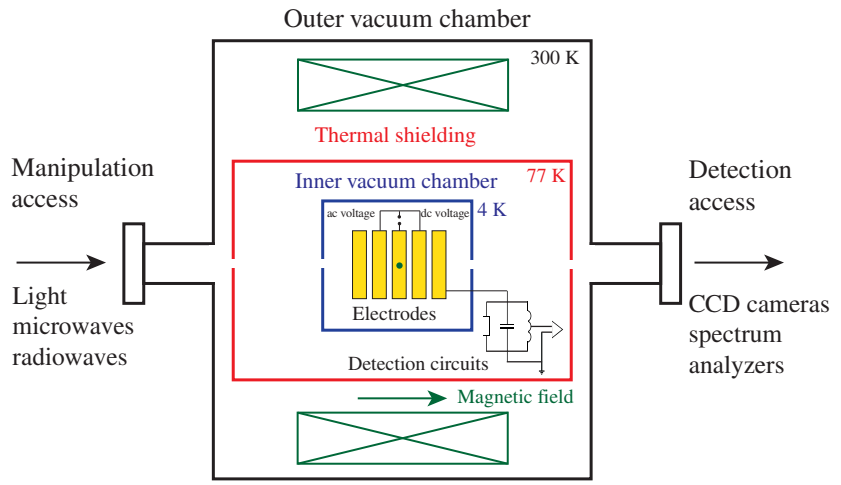

FIG. 1. Elements common to typical trap experiments. A set of electrodes supplied with ac and dc voltages is mounted in a vacuum chamber, optionally in a strong magnetic field. Cryogenic traps have, in addition, thermal shielding and cryogenic vacuum chambers at low temperatures in their surroundings. The particles are manipulated with laser beams, microwaves, and radio-frequency (rf) generators. The signals are read out by detecting image currents or fluorescent light.

\section{A. Penning traps}

In a Penning trap, there is a strong magnetic field, $B_{0}$, superimposed with an electrostatic quadrupolar potential $\Phi(z, \rho)$, which is attractive along the magnetic field axis. The motion of a charged particle in such crossed static fields is composed of three independent harmonic oscillator modes. The modified cyclotron and the magnetron modes correspond to oscillations perpendicular to the axis, while the axial mode corresponds to oscillations along the magnetic field lines. Associated with the mode oscillations are the three trap eigenfrequencies $v_{+}, v_{-}$, and $v_{z}$, respectively. Room-temperature traps such as that in Ref. [50] cool and trap ions such as ${ }^{40} \mathrm{Ca}^{+}$or ${ }^{9} \mathrm{Be}^{+}$ to the axial ground state using optical sideband cooling [51]. A delay period after cooling is followed by subsequent spectroscopy, which determines the final state of the ion, thus measuring the heating rate. The lowest heating rate achieved thus far at room temperature is reported in Ref. [50], where the increase in the number of phonons of the axial mode has been reported to be $\dot{n}=0.3 / \mathrm{s}$ with the axial frequency $v_{z}=0.3 \mathrm{MHz}$ (see Table I).

Particularly interesting for the detection of mode-energy changes are experiments dedicated to direct measurements of nuclear magnetic moments such as those of the proton [53], the antiproton [42], or ${ }^{3} \mathrm{He}^{2+}$ [54]. These experiments operate advanced cryogenic Penning-trap systems consisting of multitrap assemblies. Common to all of them is a so-called analysis trap, with a strong superimposed magnetic bottle $B(z)=B_{0}+B_{2} z^{2}$, where $B_{2}$ characterizes its strength [55]. The interaction of the magnetic bottle with the magnetic moment $\mu_{z}=\mu_{+}+\mu_{-}+\mu_{s}$ of the particle results in a magnetostatic axial energy $E_{B, z}=$ $-\mu_{z} B_{z}$, where $\mu_{+}$and $\mu_{-}$are the orbital angular magnetic moments associated with the modified cyclotron and the magnetron mode, while $\mu_{s}$ is the spin magnetic moment. As a result, the axial frequency $v_{z}=v_{z, 0}+$ $\Delta v_{z}\left(n_{+}, n_{-}, m_{s}\right)$ of the particle becomes a function of the radial trap eigenstates $n_{+}$and $n_{-}$, as well as the spin eigenstate $m_{s}$, with [52]

$$
\Delta v_{z}=\frac{h v_{+}}{4 \pi^{2} m v_{z}} \frac{B_{2}}{B_{0}}\left[\left(n_{+}+\frac{1}{2}\right)+\frac{v_{-}}{v_{+}}\left(n_{-}+\frac{1}{2}\right)+\frac{g}{2} m_{s}\right] \text {. }
$$

Measurements of single-particle magnetic moments in Penning traps rely on the detection of axial-frequency shifts $\Delta v_{z, \mathrm{SF}}$ induced by driven spin quantum transitions $\Delta m_{s}=1$. Since the nuclear magnetic moments are about 3 orders of magnitude smaller than the Bohr magneton, these experiments require the highest sensitivity with respect to the magnetic moments, which is usually achieved by the utilization of magnetic bottle strengths of the order of $B_{2} \approx 100-400 \mathrm{kT} / \mathrm{m}^{2}$. Combined with continuous measurements of the axial frequency, such strong magnetic bottles provide excellent resolution of the radial mode 
TABLE I. A list of ion traps and the relevant experimental parameters used for setting limits in this paper. The ion used, $V_{z}$, the potential barrier in the axial direction, $T_{\text {wall }}$, the temperature of the walls of the trap, and $\omega_{p}$, the fundamental frequency of the trap in the relevant direction, are listed. Also listed are $T_{\mathrm{ion}}$, the temperature of the ion in the trap, and the measured heating rate.

\begin{tabular}{lccccccc}
\hline \hline Experiment & Type & Ion & $V_{z}$ & $T_{\text {wall }}$ & $\omega_{p}(\mathrm{neV})$ & $T_{\text {ion }}(\mathrm{neV})$ & Heating rate $(\mathrm{neV} / \mathrm{s})$ \\
\hline Hite et al. [46] & Paul & ${ }^{9} \mathrm{Be}^{+}$ & $0.1 \mathrm{~V}$ & $300 \mathrm{~K}$ & $\omega_{z}=14.8$ & 14.8 & $640 \pm 30$ \\
Goodwin et al. [50] & Penning & ${ }^{40} \mathrm{Ca}^{+}$ & $175 \mathrm{~V}$ & $300 \mathrm{~K}$ & $\omega_{z}=1.24$ & 1.24 & $0.37 \pm 0.25$ \\
Borchert et al. [52] & Penning & $\bar{p}$ & $0.633 \mathrm{~V}$ & $5.6 \mathrm{~K}$ & $\omega_{+}=77.4$ & 7240 & $0.012 \pm 0.019$ \\
& & & & & $\omega_{-}=0.050$ & & \\
\hline \hline
\end{tabular}

energies with

$$
\frac{\left(\Delta v_{z}\right)}{\Delta E_{\rho}}=\frac{1}{4 \pi^{2} m v_{z}} \frac{B_{2}}{B_{0}} \approx 1 \frac{\mathrm{Hz}}{\mu \mathrm{eV}}
$$

while in axial-frequency measurements, resolutions on the order of $200 \mathrm{mHz} \cdot \sqrt{\mathrm{s}} / \sqrt{t_{\mathrm{avg}}}$ are achieved, $t_{\mathrm{avg}}$ being the averaging time, which is typically on the order of several tens of seconds. The transition rates in the radial modes $\left(d n_{ \pm}\right) / d t \propto\left(n_{ \pm} / \omega_{ \pm}\right) S_{E}\left(\omega_{ \pm}\right)$due to background noise lead to random walks in radial energy space and to axial-frequency diffusion. Here, $S_{E}\left(\omega_{+,-}\right)$is the power spectral density of a noisy background drive and $n_{+,-}$is the principal quantum number of the modified cyclotron $\left(n_{+}\right)$/magnetron $\left(n_{-}\right)$oscillator $[56,57]$.

By analyzing time sequences of axial-frequency measurements $v_{z}(t)$, the average radial quantum transition rates are obtained. With a highly optimized trap setup, with which the antiproton magnetic moment has been measured with 1.5-parts-per-billion precision [42], the Baryon Antibaryon Symmetry Experiment (BASE) experiment at CERN has reported on the observation of absolute cyclotron transition rates of 6(1) quanta per hour [52]. Together with the determination of the $n_{+}$state during the recorded measurement, this result is consistent with a projected ground state heating rate of 0.1 cyclotron quantum transitions per hour, setting an upper limit that is lower by a factor of 1800 than the best reported Paul-trap heating rates and lower by a factor of 230 than the best room-temperature Penning trap. These numbers are summarized in Table I. Note that the antiproton experiments are conducted in a background vacuum of approximately $10^{-18}$ mbar [49], constraining parasitic heating induced by collisions with background gas to a level of $4 \times 10^{-9} / \mathrm{s}$.

\section{B. Paul traps}

Paul traps, or rf traps, utilize an oscillating voltage to confine in the perpendicular direction instead of the magnetic field used for the same purpose in the Penning trap. Paul traps have a rich history of being used as mass spectrometers and, more recently, in building quantum computers [58].
The effective potential in the presence of both dc and ac potentials can be written as

$$
\psi(x, y, z, t)=\left(U_{\mathrm{dc}}+V_{\mathrm{ac}} \cos \Omega t\right) \frac{r^{2}+2\left(z_{0}^{2}-z^{2}\right)}{r_{0}^{2}+2 z_{0}^{2}} .
$$

Here, $z$ is the axial direction and $r$ is the radial direction, with the distance to the electrodes given by $r_{0}$ and $z_{0}$. The rapidly oscillating potential creates a pseudopotential for charges of both signs and this leads to an approximately simple harmonic motion close to the trap center. After laser cooling to the ground state, the total heating rate can be measured via the Raman-sideband technique [59]. There has been extensive study of the heating rate in Paul traps and its dependence on distance to electrodes, wall temperature, and trap temperature [58], as well as ionbeam treatment of electrodes [46]. Electric field noise from the electrodes has been identified as the dominant heating source, with the dependence on distance scaling as $d^{-2}$ [58] to $d^{-4}$ [60]. Although heating rates are lower for larger traps [61], smaller-sized traps employ shallower potential wells of approximately $0.1 \mathrm{~V}$. As we see, this allows mCPs with larger charge $\left(\epsilon \geq 10^{-2}\right)$ to reach the trap and hence provide sensitivity at the corresponding parameter space. Hence we reinterpret limits only from a microtrap [46]. The heating rates reported in Ref. [46] are $\dot{n}=43 / \mathrm{s}$ for the axial frequency $v_{z}=3.6 \mathrm{MHz}$. These numbers are tabulated in Table I.

\section{MILLICHARGED-PARTICLE DYNAMICS}

\section{A. Terrestrial accumulation}

If $\mathrm{mCDM}$ exists and is virialized in the galaxy, there is a flux of mCPs flowing through the Earth at all times. This mCDM stops in the atmosphere or rock overburden for large enough charge and can accumulate on Earth. This process has been treated in detail in Ref. [34] and the subsequent accumulation in Ref. [35]. We provide here a summary of the relevant results of these papers, which are used in Sec. V, and we refer the reader to Ref. [35] for details.

Following Ref. [35], we consider millicharged particles mediated by a dark photon that mixes kinematically with the SM photon. The dark-photon mass is taken to be large enough $\left(m_{A^{\prime}} \gtrsim 10^{-12} \mathrm{eV}\right)$ such that the effect of large-scale 
electric and magnetic fields can be ignored while considering $\mathrm{mCP}$ propagation [62]. In this limit, the mCPs with mass $m_{Q} \geq 1 \mathrm{GeV}$ are stuck on the Earth after thermalization [63]. The volume-averaged DM number density on Earth, $\left\langle n_{Q}\right\rangle$, can be several orders of magnitude larger than the virial DM density. It is given by

$$
\begin{aligned}
\left\langle n_{Q}\right\rangle & =\frac{\pi R_{\oplus}^{2} v_{\mathrm{vir}} t_{\oplus}}{4 / 3 \pi R_{\oplus}^{3}} n_{\mathrm{vir}} \\
& \approx \frac{3 \times 10^{15}}{\mathrm{~cm}^{3}} \frac{t_{\oplus}}{10^{10} \mathrm{y}} f_{Q} \frac{\mathrm{GeV}}{m_{Q}},
\end{aligned}
$$

where $R_{\oplus}$ and $t_{\oplus}$ are the radius and age of the Earth and $n_{\mathrm{vir}}$ and $v_{\text {vir }}$ are the galactic virial number density and velocity. However, the equilibrium-density profile is peaked at the Earth's core. This density $n_{\text {static }}$ has been calculated by taking into account the Earth's temperature and density variations in Ref. [64]. However, the sinking to the Earth's core is not immediate and there is a dynamic population, the so-called "traffic jam" density, $n_{\mathrm{t} j}$, given by

$$
n_{\mathrm{tj}}=n_{\mathrm{vir}} \frac{v_{\mathrm{vir}}}{v_{\text {term }}} \text {. }
$$

Here, $v_{\text {term }}$ is the terminal velocity in rock, given in Ref. [35]. Finally, the density in the laboratory, $n_{\text {lab }}$ is given by

$$
n_{\text {lab }}=\operatorname{Max}\left[n_{\text {static }}, \operatorname{Min}\left(n_{\mathrm{tj}},\left\langle n_{Q}\right\rangle\right)\right] .
$$

Here, we cap $n_{\mathrm{tj}}$ at $\left\langle n_{Q}\right\rangle$, since local traffic jam densities larger than average densities are unphysical due to diffusion. These results are applicable only for $\epsilon$ large enough such that the $\mathrm{mCP}$ stops in the corresponding overburden. According to Ref. [35], this is valid only for

$$
\begin{aligned}
\epsilon & \gtrsim 2 \times 10^{-4} \sqrt{\frac{m_{Q}}{\mathrm{GeV}}} \quad \text { surface }, \\
& \gtrsim 3 \times 10^{-6} \sqrt{\frac{m_{Q}}{\mathrm{GeV}}} \quad 1 \mathrm{~km} \text { mine. }
\end{aligned}
$$

It is also important to comment on the asymmetry of the $\mathrm{mCP}$ population. If the $\mathrm{mCDM}$ is a symmetric population with equal numbers of particles and antiparticles, accumulation on Earth can result in Sommerfeld-enhanced annihilations that prevent build-up. We consider here the asymmetric case such that opposite charges are carried by different species just like the SM proton and electron, such that annihilations are absent. In this scenario, for large enough $\epsilon$, the negatively charged mCPs can form deep bound states with positively charged SM nuclei, whereas the positive mCPs can only bind with the less massive electrons that also have smaller charge compared to the heavy
SM nuclei. In Ref. [35], it has been pointed out that positive mCPs with $\epsilon \geq 0.042$ bind to electrons at $300 \mathrm{~K}$ (room temperature). We find that such bound states are temporary and unstable, with electrons readily binding with the ubiquitous SM nuclei, which possess larger charge than the positive mCPs. Thus the terrestrial population of positive mCPs remains free of binding for $\epsilon \lesssim 1$ and is present as a locally thermalized population that is diffusing everywhere. Thus, the results we derive apply to all positive charges with $\epsilon \lesssim 1$ and negative charges that do not bind with nuclei, i.e., $\epsilon<\left(m_{e} / \mu_{Q, N}\right)$, where $\mu_{Q, N}$ is the reduced mass of the mCDM-nuclear system [35].

It is important to emphasize that the limits put on the ambient number density are applicable to mCPs charged directly under the SM photon as well as those mediated by a dark photon as long as the dark-photon mass is below the relevant momentum transfer for scattering with ions, which is around $1 \mathrm{eV}$.

\section{B. Passage through apparatus}

We next turn to the trajectory of mCPs through the trap peripherals in order to reach the trapped ion. We need to know the density and temperature of the mCPs reaching the ion. In this subsection, we explain the main factors entering the calculation, but as the calculation itself is somewhat involved we leave the details to Appendix A. Our main result is in Eq. (A9), relating the number density of mCPs at the position of the ion, $n_{\text {ion }}^{Q}$, to the ambient number density on the Earth, $n_{\text {lab }}$. This is the equation we use to set our limits on mCPs.

At equilibrium, mCPs are expected to have roughly uniform density near the Earth's surface, including permeating all materials. However, the conditions of the experiment can affect this naive expectation for several reasons.

First, some of the ion traps we consider are cryogenic. Over essentially all of our parameter space, the mCPs have a short interaction length in material and so will rapidly thermalize to the cryostat temperature as they enter the experiment. By itself, this would lead to an increase in $\mathrm{mCP}$ density by a factor inversely proportional to the $\mathrm{mCP}$ velocity $(\propto 1 / \sqrt{T})$, because the fluxes entering and leaving the cryostat must be equal in equilibrium.

Second, the ion traps are surrounded by metal that has a work function that can affect the passage of mCPs. This is only relevant for mCPs of relatively large charge $(\epsilon \gtrsim$ $10^{-2}$ ) but for those it can be a significant effect. The work function for mCPs, which we call $\epsilon \phi$, is not simply $\epsilon$ times the work function for electrons. For electrons, the work function arises from several contributions of varying sign including, for example, the binding to the lattice of nuclei, the Fermi sea of other electrons, and surface effects such as the "double layer" or the image-charge potential. Several of these do not apply or are negligible for mCPs. Recall 
that we are only considering positively charged mCPs, since the negative ones may be stuck deeply bound to some nucleus somewhere on the Earth. Thus the main effects are repulsion by the double layer and possibly also repulsion from the nuclei. We consider the work function for mCPs in more detail in Appendix 2. Our conclusion is that the work function is repulsive for positively charged mCPs and thus every metal sheet provides a barrier for $\mathrm{mCPs}$ to cross. The size of the potential energy barrier that has to be crossed is $\phi \sim$ a few $\mathrm{eV}$. Note that for an experiment at room temperature $(T \sim 0.03 \mathrm{eV})$, the metal barriers are then irrelevant for charges $\epsilon \lesssim 10^{-1}$ because the Boltzmann tail easily pushes a fast enough rate of mCPs over the barrier. For a cryogenic experiment at $T \sim 6 \mathrm{~K} \sim 5 \times 10^{-4} \mathrm{eV}$, the metal barrier will be relevant for charges $\epsilon \gtrsim 10^{-3}$ and essentially insurmountable for charges $\epsilon \gtrsim 10^{-2}$. As we show in Appendix A, the most important effect comes from an experiment encased in two different metals, where the work function for mCPs rises from the outer metal to the inner metal. We take this difference to be $\Delta \phi=3 \mathrm{eV}$ for all the experiments we consider, since this is a conservative estimate, as we show in Appendix 2.

Third, the ions are always in a region of ultrahigh vacuum. This means that pumps are used to remove the SM particles. Given a short interaction length of the $\mathrm{mCP}$ in materials, these pumps could remove the mCPs from the ion chamber as well. In one of the experiments we consider (Goodwin et al. [50]), this effect is not relevant because the trap is at room temperature and the region of sensitivity is at low enough $\epsilon$ that the millicharges pass easily through the walls [65]. In the other two experiments we consider (Hite et al. [46] and Borchert et al. [52]), the vacuum pumps are turned off well before the actual data taking is begun. In the case of Ref. [52], this is at least a year, while for Ref. [46], we conservatively assume that it is only a day [66]. Since mCPs are always continually flowing in from the walls of the vacuum chamber, this would rapidly refill the trap region (in $\mathcal{O} \mu s$ for gigaelectronvolt masses and $5 \mathrm{~K}$ temperature) and this effect would not be relevant, except for the largest charges, where the refill can be slow because of the work function of the surrounding metal. This does mean that, depending on the parameters of the $\mathrm{mCP}$ and of the ion trap, the number density in the trap may be either in the equilibrium regime or in the filling regime. This is why Eq. (A8) has two different regimes. The number density of mCPs inside the trap $n_{\text {trap }}$ is related to the ambient number density on the Earth $n_{\mathrm{lab}}$ in Eq. (A8). There is then one remaining step to find the number density of mCPs at the position of the ion.

Fourth, the ion trap itself has applied electromagnetic fields to trap the ion. These can affect the passage of the
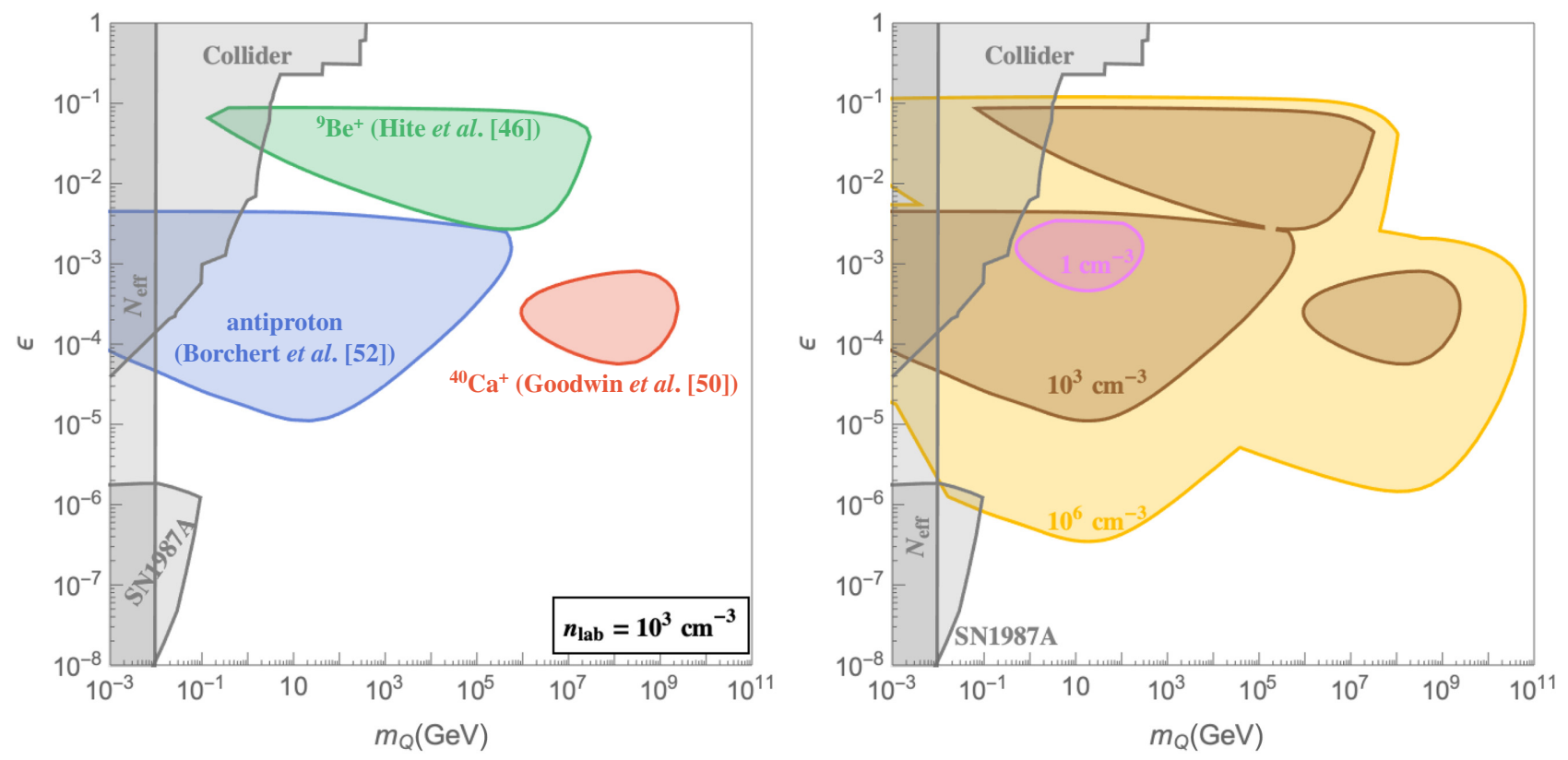

FIG. 2. A compilation of new limits [the shaded areas are where the parameters are excluded at the $>95 \%$ confidence limit (CL)] using existing heating measurements from various traps in Table I: a room-temperature Paul trap, from Hite et al. [46] ( $\omega_{z}$ mode); a room-temperature Penning trap, from Goodwin et al. [50] ( $\omega_{z}$ mode); and a cryogenic Penning trap, from Borchert et al. [52] ( $\omega_{-}$ mode). Left: a comparison between traps for a benchmark ambient density $n_{\text {lab }}=10^{3} \mathrm{~cm}^{-3}$. Right: combined limits from the three traps for different $n_{\text {lab }}$. Limits on the virial dark matter fraction are shown in Fig. 4. Existing limits on mCPs (with no assumption on the DM fraction) are shown in gray. The collider limits are obtained from Refs. [2-7], the SN1987A limits from Ref. [13], and the big bang nucleosynthesis $N_{\text {eff }}$ limits from Ref. [15]. 
$\mathrm{mCPs}$, though again this is only relevant for larger charges $\epsilon \gtrsim 10^{-3}$. Table I lists parameters of the various experiments that we consider. All the experiments use an electric dc potential to confine the ion in the axial direction. The height of the potential barrier along the axial direction is listed as $V_{z}$. The mCPs in the trap are thermalized to the temperature of the walls of the trap, $T_{\text {wall }}$. Starting from the number density of mCPs calculated inside the trap, $n_{\text {trap }}$ in Eq. (A8), we take a Boltzmann suppression on the number density that can make it up the axial barrier height. Thus we take the final number density at the position of the ion to be given by Eq. (A9). For the experiments, we consider that this Boltzmann suppression is relevant for setting the ceiling (largest $\epsilon$ values) of the Goodwin regions in Fig. 2, but is irrelevant for the other regions. The Penning traps use a magnetic field to confine the ions in the radial direction. This $B$ field will cause mCPs with a large enough charge to circle around the axial $B$-field lines but they are still free to move along the axis. The magnetic field does not change the phase-space density of the mCPs and so it does not significantly affect our signal. The Paul traps use an rf potential to confine the ion in the radial direction. This potential around the minimum is locally attractive for the positive $\mathrm{mCP}$, independent of the sign of the charge of the trapped ion. An mCP coming from far outside the rf fields in a radial direction could give a barrier in principle. However, for mCPs approaching along the axial direction, the rf pseudopotential in the axial direction is weak and negligible, and then such mCPs will actually be concentrated in the radial direction, toward the ion at the center of the trap, since the potential is locally attractive. We conservatively ignore this possible (Sommerfeld-like) enhancement, though it could be large. For large enough mCP masses, $m_{Q} \gtrsim 10^{10} \mathrm{GeV}$, the freefall under gravity can generate velocities much larger than the thermal velocities assumed. This can increase the heating rate further. We conservatively ignore this effect and leave its consideration for future work.

\section{OBSERVABLES FOR MILLICHARGED PARTICLES IN ION TRAPS}

In this section, we consider the interaction of ambient mCPs with ion traps to identify observables for detection. As seen in Sec. III B, the mCPs enter the ion trap with effective temperature $T_{Q} \geq T_{\text {wall }}$, where $T_{\text {wall }}$ is the wall temperature. $T_{\text {wall }}=5.6 \mathrm{~K}$ for the cryogenic trap that we consider [52] and $T_{\text {wall }}=T_{\text {room }} \approx 300 \mathrm{~K}$ for roomtemperature traps. The ions in the trap are at a much colder temperature $T_{\text {ion }} \ll T_{\text {wall }}$. With this hierarchy of temperatures, the $\mathrm{mCPs}$ can cause two types of signals.

The first signal involves individual scattering events that impart energy $E_{\text {ion }}$ to the ion, thus leading to a change in its harmonic oscillator quantum number. This signal is similar to dark matter scattering in a conventional dark matter detector. The rate of events has to be slower than the rate at which the ions are interrogated in order to be registered as single events. $E_{\text {ion }}$ also needs to exceed the energy resolution $E_{\text {res }}$ for detection.

The second type of signal is the heating of the trapped ion due to collisions with multiple mCPs. In this case, the individual hits $E_{\text {ion }}$ can be smaller than $E_{\text {res }}$ and only the sum needs to exceed this resolution.

For both of these signal types, we only consider individual energy transfers $E_{\text {ion }}$ much larger than the typical energy spacing of the trap $\omega$, for concreteness $E_{\text {ion }} \geq 10 \omega$ [67]. Such large energy transfers correspond to momentum transfers $q r \gg 1$, where $r$ is the radius of the cyclotron or magnetron motion. This is in contrast to decay or excitation due to photonic emission or absorption or electrode noise where $q=\omega$, so that $q r \ll 1$. As a result, the selection rules that apply in the dipole approximation $q r \ll 1$ do not apply to scattering. Furthermore, the large momentum transfer is inherently short distance in nature. factorizing from the influence of the electromagnetic fields of the trap. Hence, we can use the free-particle approximation. In this limit, the trapped ion can be approximated as a free particle with initial energy $T_{\text {ion }}$ and final energy $T_{\text {ion }}+E_{\text {ion }}$. Equivalently, for energy transfers much larger than the ionenergy spacing in the trap, the form factor that incorporates the wave-function overlap can be approximated to unity.

We start to quantify both of these signals by the angular differential cross section given by the Rutherford formula,

$$
\frac{d \sigma}{d \Omega}=\frac{2 \pi \alpha^{2} \epsilon^{2}}{\mu^{2} v_{\mathrm{rel}}^{4}(1-\cos \theta)^{2}},
$$

where $\theta$ is the scattering angle, $\mu$ is the reduced mass, and $v_{\text {rel }}$ is the relative velocity. We next introduce kinematic variables that simplify the computation of the scattering rate. The results are presented here, with the details of the derivation described in Appendix B. The incoming mCP and trapped-ion velocities are assumed to be $\mathbf{v}_{\mathbf{Q}}$ and $\mathbf{v}_{\text {ion }}$, respectively. The center-of-mass (c.m.) velocity $\mathbf{v}_{\mathbf{c} . \mathbf{m} \text {., is }}$ given by

$$
\mathbf{v}_{\mathbf{c} . \mathbf{m} .}=\frac{\left(m_{\text {ion }} \mathbf{v}_{\text {ion }}+m_{Q} \mathbf{v}_{\mathbf{Q}}\right)}{m_{\text {ion }}+m_{Q}} .
$$

The change in velocity of the ion, $\Delta \mathbf{v}_{\text {ion }}$, is given by

$$
\begin{aligned}
\Delta \mathbf{v}_{\text {ion }}= & \frac{m_{Q}}{m_{\text {ion }}+m_{Q}}\left[(\cos \theta-1)\left(\mathbf{v}_{\text {ion }}-\mathbf{v}_{\mathbf{Q}}\right)\right. \\
& \left.+\sin \theta\left|\mathbf{v}_{\text {ion }}-\mathbf{v}_{\mathbf{Q}}\right| \mathbf{n}_{\perp}\right] .
\end{aligned}
$$

The transferred energy, $E_{\text {ion }}$, is given by

$$
E_{\text {ion }}=m_{\text {ion }} \mathbf{v}_{\mathbf{c} \cdot \mathbf{m} .} \cdot \Delta \mathbf{v}_{\text {ion }} .
$$


Given a threshold $E_{\mathrm{thr}}$, the single-event rate $R_{\text {single }}$ with energy transfer $E_{\text {ion }}$ above this threshold is

$$
\begin{aligned}
& R_{\text {single }}\left(E_{\text {ion }} \geq E_{\text {thr }}\right) \\
& =n_{\text {ion }}^{Q} \int d^{3} \mathbf{v}_{\mathbf{Q}} g_{Q} \int d^{3} \mathbf{v}_{\text {ion }} g_{\text {ion }} \\
& \quad \times \int d \Omega\left|v_{Q}-v_{\text {ion }}\right| \frac{d \sigma}{d \Omega} \Theta\left(\left|E_{\text {ion }}\right|-E_{\text {thr }}\right) .
\end{aligned}
$$

Here, $n_{\text {ion }}^{Q}$ is the number density of mCPs at the ion position given by Eq. (A9) and $g_{\text {ion }(Q)}$ is the Maxwell-Boltzmann distribution for the ion or $\mathrm{mCP}$ with temperatures $T_{\text {ion }}$ and $T_{\text {wall }}$, respectively.

The heating rate per ion, $\dot{H}$, can be computed through

$$
\begin{aligned}
\dot{H}= & n_{\text {ion }}^{Q} \int d^{3} \mathbf{v}_{\mathbf{Q}} g_{Q} \int d^{3} \mathbf{v}_{\text {ion }} g_{\text {ion }} \int d \Omega\left|v_{Q}-v_{\text {ion }}\right| \frac{d \sigma}{d \Omega} \\
& \times E_{\text {ion }} \Theta\left(\left|E_{\text {ion }}\right|-E_{\text {thr }}\right) \Theta\left(E_{\text {samp }}-\left|E_{\text {ion }}\right|\right) .
\end{aligned}
$$

The Heaviside theta function ensures the inequality $E_{\text {thr }} \leq$ $E_{\text {ion }} \leq E_{\text {samp. }}$. Here, $E_{\text {samp }}$ is defined so as to prevent the average heating rate from including contribution from extremely rare events. It is defined through

$$
R_{\text {single }}\left(E_{\text {ion }} \geq E_{\text {samp }}\right) t_{\text {obs }}=1,
$$

where $t_{\mathrm{obs}}$ is the total observation time.

\section{RESULTS}

In this section, we set constraints on mCPs and make projections for the future by using the expressions for the signal rates derived in Sec. IV.

\section{A. Limits from existing measurements}

To obtain limits from existing measurements, we use the data presented in Table I. All of the trap parameters for the ${ }^{9} \mathrm{Be}^{+}$trap are taken from Ref. [46], while the trap depth is conservatively taken to be $V_{z}=0.1 \mathrm{~V}$, an order of magnitude larger than the typical potential depths in microtraps [68]. While the rest of the parameters for the ${ }^{40} \mathrm{Ca}^{+}$experiment are provided in Ref. [50], we obtain the potential depth $V_{z}=175 \mathrm{~V}$ from the authors. Finally, for Ref. [52], we use the parameters $T_{\text {wall }}=5.6 \mathrm{~K}$ and $V_{z}=0.6 \mathrm{~V}$. The analysis in Ref. [52] deals with measuring heating of the cyclotron mode $\left(\omega_{+}\right)$, which has been observed to be a random-walk process. mCPs will preferentially heat the magnetron mode $\left(\omega_{-}\right)$due to the smaller momentum transfer required for Rutherford scattering and this presents as a dc heating rate. Reanalysing existing data that have been partly presented in Ref. [52], we find a dc heating rate limit $\dot{H}=0.012 \pm 0.019 \mathrm{neV} / \mathrm{s}$.

We start by plotting $95 \%$ confidence limit limits derived from existing heating measurements in Fig. 2 in the millicharge $\epsilon$ versus mass $m_{Q}$ parameter space for contours of constant ambient density $n_{\text {lab }}$. As mentioned earlier, for $\mathrm{mCDM}$, the laboratory density is expected to be orders of magnitude larger than the virial density, i.e., $n_{\mathrm{lab}} \gg n_{\mathrm{vir}}$. In the left panel, we show limits arising from the antiproton trap [52] in blue, ${ }^{40} \mathrm{Ca}^{+}$[50] in red, and ${ }^{9} \mathrm{Be}$ in green [46] for an ambient density of $n_{\text {lab }}=10^{3} / \mathrm{cm}^{3}$. The dominant limits arise from the antiproton trap owing to its superior heating rate, as seen in Table I. However, since it is a cryogenic trap, the limits disappear at $\epsilon \approx 10^{-2}$, owing to the suppression arising from $\mathrm{mCPs}$ no longer penetrating metals due to their work function. As explained in Sec. III and Appendix A, this factor roughly scales as $e^{-\left(\epsilon \Delta \phi / T_{\text {wall }}\right)}$, where $\Delta \phi$ is the difference in work functions between two adjacent metals. Hence this suppression is ameliorated for room-temperature traps such as the ${ }^{9} \mathrm{Be}^{+}$trap [46] in green, which extends to $\epsilon \approx 0.1$. While the ${ }^{40} \mathrm{Ca}^{+}$trap in red is also at room temperature, the trap is at a potential of 175 $\mathrm{V}$ and thus mCP charges above $\epsilon \approx 10^{-3}$ do not reach the ion. However, there is reach to higher $\mathrm{mCP}$ masses for this trap as a result of the ion being more massive. The right panel of Fig. 2 corresponds to combined limits from these three experiments for different $n_{\text {lab }}$.

As is clear from both figures, existing data for anomalous heating in traps set world-leading bounds on mCPs thermalized locally. The bounds are applicable to orders of magnitude in the mCP mass $m_{Q}$ as well as many orders of magnitude in charge. Number densities as small as $n_{\mathrm{lab}}=1 \mathrm{~cm}^{-3}$ are ruled out around the $\epsilon \approx 10^{-3}$ and $m_{Q} \approx$ $10 \mathrm{GeV}$ parameter point.

In order to compare these limits on the ambient $\mathrm{mCP}$ population those that already exist in the literature, we fix the $\mathrm{mCP}$ mass at $m_{Q}=10 \mathrm{GeV}$ and show limits in the $n_{\text {lab }}$ versus $\epsilon$ plane in Fig. 3. The same color coding is followed as in Fig. 2. In gray, we show limits from LEP [7] as well as limits on mCPs bound in matter arising from oil-drop [36] and levitation experiments [37,38]. As noted earlier, the limits on mCPs bound in matter are applicable only to negative mCPs with a large enough charge such that binding with SM nuclei is possible. Furthermore, if $\mathrm{mCP}-$ SM bound states exist, there is no guarantee that these bound states will be evenly distributed all over the Earth. However, for the positive mCPs, none of these caveats apply and they thermalize and distribute themselves over the entire volume of the Earth. Regardless, as seen in Fig. 3, the limits obtained from ion traps are orders of magnitude stronger than those from the levitation experiments. For $\epsilon \approx 3 \times 10^{-3}$, laboratory densities as small as $n_{\text {lab }} \gtrsim 1 \mathrm{~cm}^{-3}$ are ruled out by the measured heating rate at the antiproton experiment [52].

Next, in Fig. 4, we convert limits on $n_{\text {lab }}$ into limits on the fraction of virial DM existing in $\mathrm{mCPs}, f_{Q}=$ $\left(\rho_{Q} / \rho_{\text {DM }}\right)$. For this purpose, we use $n_{\text {lab }}$ from Eq. 6 . Existing limits from colliders are shown in gray. The solid parts of the colored contours correspond to the region where the incoming virial DM gets thermalized within $1 \mathrm{~m}$ and hence 


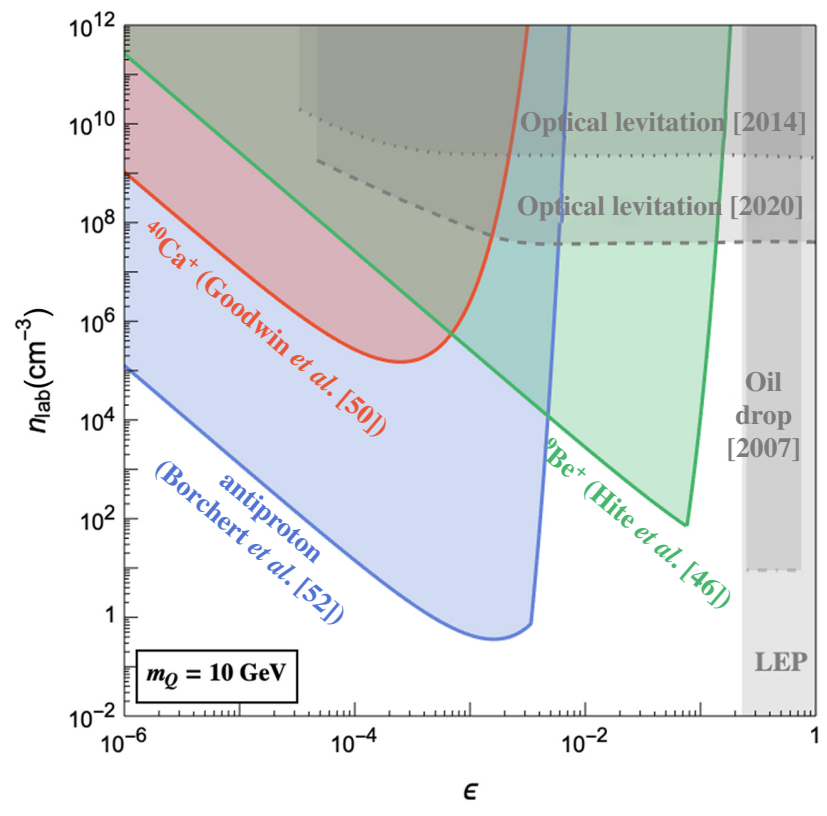

FIG. 3. A comparison of the (95\% confidence limit) limits derived in this work from Refs. [46,50,52] with existing limits from oil-drop [36] and levitation [37,38] experiments and LEP [7] for $m_{Q}=10 \mathrm{GeV}$.

the robust current limits that we set are restricted to this region, i.e., above the top black line. The dashed lines show the reach for an identical heating-rate experiment that is

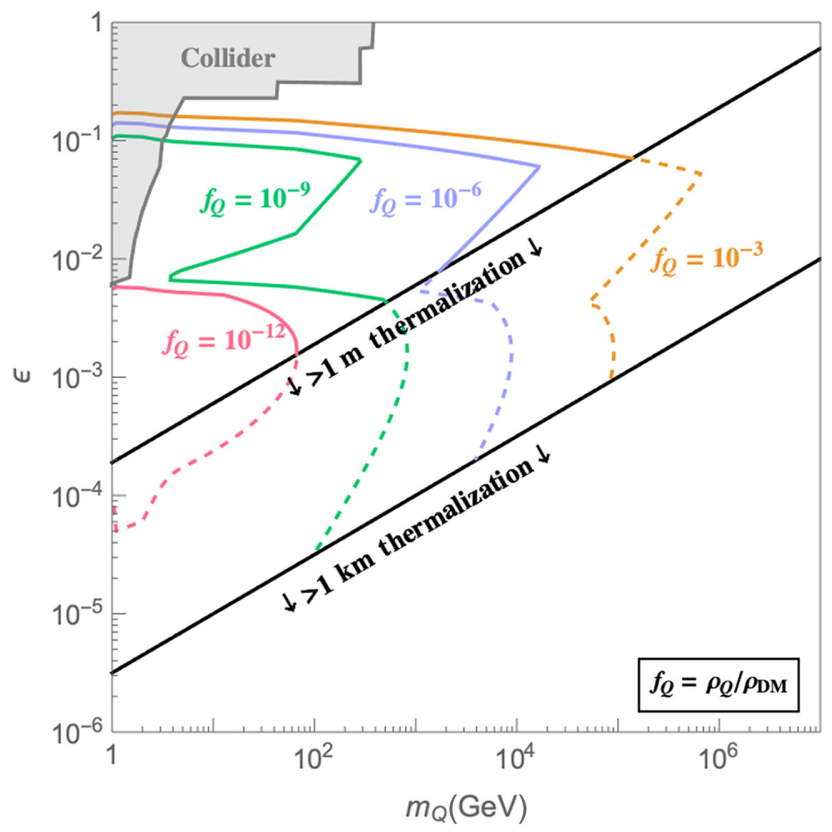

FIG. 4. The limits and projections (95\% CL) on the virial DM fraction in mCPs as a function of $\epsilon$ and $m_{Q}$. Since the current experiments are all conducted at the surface, the robust limits are only above the "> $1 \mathrm{~m}$ thermalization" line, where the colored contours are solid. For a hypothetical deep-mine experiment, the dashed part of the contours is also accessible. conducted in a deep mine at a depth of $1 \mathrm{~km}$. Virial DM fractions as small as $f_{Q} \approx 10^{-12}$ are already ruled out using existing heating data for DM masses in the $1-10 \mathrm{GeV}$ mass range. For heavier masses, the terminal velocity is larger and hence the traffic jam densities are smaller. Nonetheless, we set limits on DM fractions as small as $f_{Q}=10^{-3}$ for masses as large as $m_{Q} \approx 10^{5} \mathrm{GeV}$.

The limits presented above are derived using data from existing experiments that measure the anomalous heating rate. We next make projections for the future to capture improvements in reducing the heating rate as well as to incorporate specific modifications designed for $\mathrm{mCP}$ detection.

\section{B. Projections}

The limits shown thus far arise from the cumulative heating rate measured. Another promising avenue is the (non)observation of individual event rates. In Fig. 5, we compare projections from a nonobservation of single events with $E_{\text {ion }} \geq 10 \omega_{+}$, with the same parameters as existing data in Ref. [52] in dark blue, with the heating of the much smaller $\omega_{-}$, in brown, for $n_{\text {lab }}=10^{3} \mathrm{~cm}^{-3}$.

We find that this projection is near identical to the heating limit shown in brown. Both the heating limits as well as the event-rate sensitivity are expected to improve in the future. For instance, heating rates are known to decrease with a larger electrode distance or an increase in the frequency of the trapped ion [58]. Whereas the $\mathrm{mCP}$ search with the heating rate in the current BASE apparatus is already background limited, the event-rate analysis is not.

It is unclear what the limiting background will be for events with $E_{\min }=10 \omega$. The harmonic oscillator selection rules prevent excitation of $E_{\text {ion }} \gg \omega$ due to black-body radiation and electrode noise [56]. Background gas particles at the existing pressure of $3 \times 10^{-18}$ mbar and $100 \AA^{2}$ cross section correspond to one event every 5000 years. In Fig. 5, we make projections for various experimental choices assuming event rates of $1 \mathrm{yr}^{-1}$ for $n_{\text {lab }}=$ $10^{3} \mathrm{~cm}^{-3}$. In pink, we show projections for a trapped proton, keeping the existing energy threshold $E_{\min }=10 \omega_{+}$ and other parameters as in Ref. [52]. Next, we explore the reach for highly charged ions as well as ions in a lattice (see, e.g., Ref. [69] for recent heating limits from highly charged ${ }^{40} \mathrm{Ar}^{13+}$ ions in a lattice). In light blue, we consider the same setup as in Ref. [52] but we consider the reach for hydrogenlike calcium $\left(\mathrm{Ca}^{19+}\right)$, which enhances the Rutherford-scattering cross section due to the large ionic charge. This limit is also equivalent to that obtained with 361 ions in a Coulomb crystal (see, e.g., Ref. [70]), with its center-of-mass mode observed for 1 year. In green, we show the effect of a vast improvement in sensitivity to the energy jump of a single event, $E_{\min }=10 \omega_{-}$, envisioned in the future. 


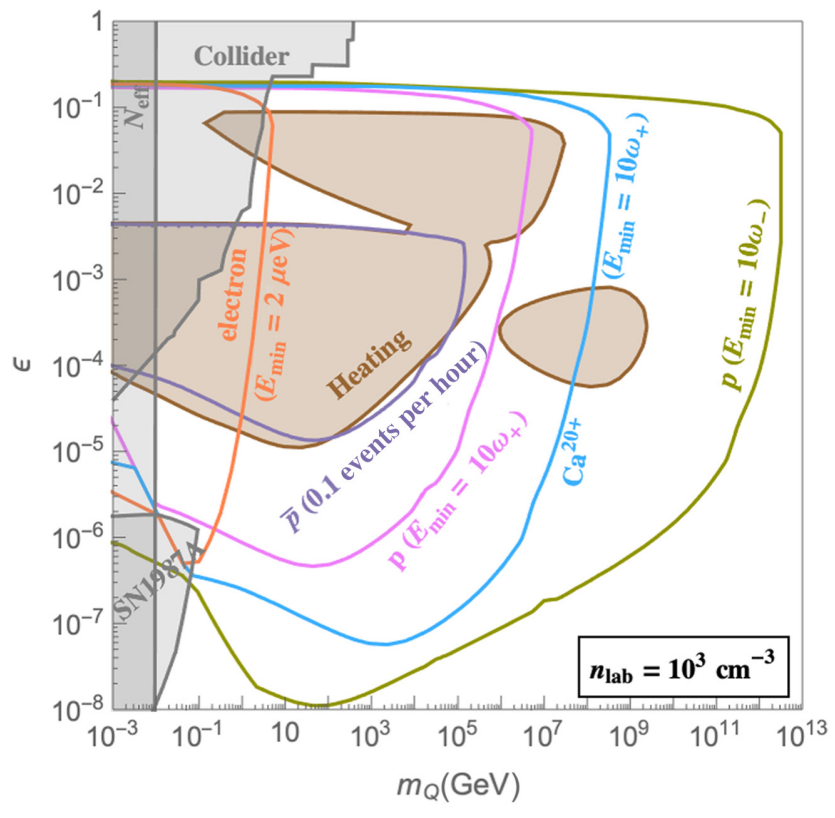

FIG. 5. Event-rate limits and projections for $n_{\mathrm{lab}}=10^{3} \mathrm{~cm}^{-3}$. Existing limits from heating of the $\omega_{-}$mode are shown (brown shaded), from Fig. 2. The projection for a search for single events in the BASE experiment [52] with an energy deposit above $10 \omega_{+}$and a rate of 0.1 event per hour are shown (dark blue). Here, $\omega_{+}=77.4 \mathrm{neV}$ and $\omega_{-}=0.050 \mathrm{neV}$. Next, projections are also shown assuming sensitivity to one-event-per-year event rates. In pink, we show the sensitivity from the existing setup of Ref. [52]. The light blue curve corresponds to swapping a single (anti)proton with hydrogenlike calcium or, equivalently, trapping 361 calcium ions in a Coulomb crystal and measuring its centerof-mass mode. We also show the reach for a futuristic experiment conducted with protons with energy thresholds of $E_{\min }=10 \omega_{-}$ in green. Finally, the reach from a hypothetical electron trap with trap frequency $\omega=200 \mathrm{neV}$ and $E_{\min }=2 \mu \mathrm{eV}$ is shown in orange.

Finally, we consider a hypothetical electron trap, with trap frequency $\omega=0.3 \mathrm{GHz}$ and $E_{\min }=3 \mathrm{GHz}$. Despite the large trap frequency required for electron trapping, it is competitive with ions in the small- $m_{Q}$ regime. This is because the momentum transfer is much smaller for electron targets compared to ions for the same energy transfer. It is important to note that, currently, electron trapping has been demonstrated in the $>100 \mathrm{GHz}$ range [39] but these systems typically employ wall temperatures lower than the level spacing in order to avoid heating due to black-body radiation. However, for thermalized mCPs, we require the wall temperature to exceed the level spacing, i.e., $T_{\text {wall }} \geq \omega$. This configuration has been demonstrated recently in Ref. [71]. However, in a trap with a wall temperature greater than the level spacing, there would generally be too high a scattering rate from black-body photons to make a sensitive detector [72]. Therefore, current electron traps likely do not set competitive limits but future improvements may turn these into sensitive $\mathrm{mCP}$ detectors.

It has also been argued in Ref. [47] that ion traps are ideal $\mathrm{mCP}$ detectors. We obtain different quantitative results for projected sensitivities, including the presence of finite high-mCP-mass thresholds. According to our best understanding, the discrepancy arises from our consideration of the ion velocity in the scattering process and the use of a different model for the local mCP density. For the $\mathrm{mCP}$ density, we use a model that incorporates the terrestrial accumulation of mCPs, the effect of work functions, realistic trapping potentials, and mean-free paths that result in a maximum $\mathrm{mCP}$ charge above which there is no reach, among other changes to the sensitivity curves.

\section{Discovery potential}

If a signal is observed, how do we confirm that it has a DM origin? As explained earlier, the only known SM process that can cause the single-event signal with $E_{\text {ion }} \gg \omega$ is due to collision with background gas but this is estimated to be one event per ion every 5000 years. As a result, its observation is strongly suggestive of an origin beyond the SM. The heating rate, on the other hand, is already observed and thus is only used to set a limit at present. Next, we comment on specific discrimination strategies.

Signal discrimination requires the ability to adjust the mCP density in the ion trap $n_{\text {ion }}^{Q}$, which depends, among other parameters, on the trapping voltage $V_{z}$, the wall temperature $T_{\text {wall }}$, and the laboratory $\mathrm{mCP}$ density outside the ion trap, $n_{\text {lab }}$ (see Appendix A). Changing $T_{\text {wall }}$ and $V_{z}$ can achieve a change in $n_{\text {ion }}^{Q}$; however, these either impact the operation of the ion trap itself or change the ion frequency, so that further discrimination of electric field noise at the modified ion frequency would be required. A promising detection concept is therefore to modify $n_{\text {lab }}$ in the iontrap environment. Ion experiments have been shown to be transportable [73-75] and can thus be conducted at different altitudes: experiments conducted deep underground in mines, at high altitudes, or in space will have drastically different $\mathrm{mCP}$-induced heating rates due to the large densities in mines and the virial densities in space. Furthermore, $n_{\text {lab }}$ can be controlled in situ by enclosing the ion trap in a double-walled high-voltage platform where the inner wall is set on a potential $U_{\mathrm{HV}}$. mCPs scatter at thermal energies with the air between the two walls, so that the electric field modifies the $\mathrm{mCP}$ density on the repelling surface by a Boltzmann factor $\exp \left(-\epsilon U_{\mathrm{HV}} / k_{B} T_{\text {room }}\right)$ compared to the attracting wall. Consequently, we can enhance the $\mathrm{mCP}$ density for detection by placing the ion trap on an attractive potential and discriminate potential signals from electric field noise by means of a repelling potential. The advantage of this approach is that the potential change is independent of the ion-trap operation and the voltage range is much higher, e.g., on the order of $10 \mathrm{kV}$ for a platform in 
a university laboratory, or even approximately $100 \mathrm{kV}$ for a dedicated high-voltage platform [76,77], in comparison to $10 \mathrm{~V}$ in changing $V_{z}$ or $1 \mathrm{kV}$ floating the trap potential. This can be used as a viable background discrimination tool even for mCPs with $\epsilon \sim 10^{-5}$ to $10^{-6}$.

\section{DISCUSSION AND CONCLUSIONS}

Generic cosmologies should produce nontrivial abundances of well-motivated stable particles that make up some or all of the observed DM density provided that the reheat temperature is high enough. Such a cosmic density of millicharged particles, with a large enough charge, can get stopped on Earth and can accumulate through the history of the planet, forming an overdense locally thermalized population. In this work, we analyze the utility of ion traps as detectors of such an mCP population. Ions trapped in harmonic potentials can detect energy deposits as small as the nanoelectronvolt range, with low intrinsic backgrounds. We show that the existing measurement of heating rates in Penning and Paul traps [46,50,52] sets strong limits on a wide range of $\mathrm{mCP}$ masses and charges, as seen in Fig. 2. These limits on the ambient thermalized population improve on existing limits from levitated spheres by several orders of magnitude, with no assumptions about the binding of mCPs in material. This can be seen in Fig. 3.

These limits can, in turn, be interpreted as limits on the fraction of virial DM existing in mCPs, $f_{Q}=\rho_{Q} / \rho_{\mathrm{DM}}$. We find in Fig. 4 that fractions as small as $f_{Q} \gtrsim 10^{-12}$ are ruled out for masses around $1-100 \mathrm{GeV}$ for millicharge around $10^{-3}$. Smaller charges can be probed with a similar setup conducted deep underground.

Turning to future prospects, while modest improvements in the observed heating rates are expected, greater strides can be made with single-event observation, as seen in Fig. 5. With event per year sensitivities, a single (anti)proton can improve upon the existing bounds from heating by one order of magnitude with energy thresholds of approximately $100 \mathrm{neV}$. Reduction of the energy threshold can further increase the parameter space that can be probed.

Another viable direction is to use a single highly ionized heavy ion [69] that increases the Rutherford cross section with mCPs. Multiple ions that form a Coulomb crystal can also result in increased sensitivity. While we make projections only for the single-event rate for a Coulomb crystal, it is reasonable to expect better signal-background discrimination by considering in detail the selection rules for collective excitations of the crystal. Heavier ions will, however, require improvements in energy resolution in order to be sensitive to the same quantum jump as an (anti)proton experiment. Finally, electrons can be stably trapped in deep potential wells. Due to its lower mass, an electron can extract approximately $m_{p} / m_{e}$ more energy than a proton for the same momentum transfer. For masses around $1 \mathrm{GeV}$ and below, electron traps might be a promising alternative to probe low charges. It is important to emphasize the complementarity of different traps. Existing traps of larger sizes have lower heating rates but feature deeper potentials. Hence, they are suited for probing small charges, whereas microtraps are ideal for larger charges. The choice of the trapped charged SM particle - a heavy ion, a proton, or an electron - can provide optimal sensitivity to different masses due to kinematic matching. For $\mathrm{mCP}$ detection purposes, a large trap with shallow trapping potentials with resolution to a small number of quantum jumps would be optimal.

Finally, we mention another possible direction for $\mathrm{mCP}$ searches. Storage rings for partially stripped ions [78] may be thought of as ion traps with many features that can be exploited for $\mathrm{mCP}$ searches, including ultrahigh vacuum, demonstrated long lifetimes (up to days) of large quantities of stored ions, ion-cooling techniques, etc. Although these experiments may require challenging systematic studies, there are two possible effects that could be explored for $\mathrm{mCP}$ searches: (1) scattering of the ions on the mCPs present in the storage ring (similar ideas for contact interactions with thermalized DM with LHC beam lifetime are explored in Ref. [64]); and (2) ionization of partially stripped ions due to collisions with mCPs. For the latter, storage rings with low-charge ions [79] may be most attractive.

Ion trapping has myriad applications, including the realization of qubits for a quantum computer. There are significant resources invested in this endeavor, which should translate into longer stability, reduced heating rates, and scaling to large numbers of ions, as well as the realization of low backgrounds in electron traps. It is exciting that these improvements translate directly into increased sensitivity of dark matter detection.

\section{ACKNOWLEDGMENTS}

We would like to thank Richard Thompson for useful discussions regarding the particulars of the experimental data in Ref. [50]. The work of D.B. and F.S.K. was supported by the Cluster of Excellence "Precision Physics, Fundamental Interactions, and Structure of Matter" (PRISMA+ EXC 2118/1) funded by the German Research Foundation (DFG) within the German Excellence Strategy (Project ID 39083149), by Grant No. European Research Council (ERC) under the European Union Horizon 2020 research and innovation program (Project Dark-OST, Grant Agreement No. 695405), and by the DFG Reinhart Koselleck project. S.U. acknowledges support by RIKEN and the Max Planck-RIKEN-Physikalisch-Technische Bundesanstalt (PTB) Center for Time, Constants and Fundamental Symmetries. C.S. acknowledges support by the ERC (Project Symmetry Tests in Experiments with Portable Antiprotons, Grant Agreement No. 852818) and 
the Institute of Physics in Mainz. P.W.G. and H.R. acknowledge support from the Simons Investigator Award 824870, U.S. Department of Energy (DOE) Grant No. DE-SC0012012, the Grant No. PHY-2014215, the DOE High Energy Physics (HEP) Quantum Information Science Enabled Discovery (QuantISED) Award No. 100495, and the Gordon and Betty Moore Foundation Grant No. GBMF7946. This work was also supported by the U.S. Department of Energy, Office of Science, National Quantum Information (NQI) Science Research Centers through the Fermilab Superconducting Quantum Materials and Systems (SQMS) NQI Center.

\section{APPENDIX A: PASSAGE THROUGH TRAP}

In this appendix, we provide the details of the passage through the trap, as is relevant to the discussion in Sec. B. The aim is to relate the ambient density in the laboratory $n_{\text {lab }}$ to the density at the point where the ion is trapped, $n_{\text {ion }}^{Q}$.

\section{Number density of mCPs in trap}

Common to both Penning and Paul traps is the metallic high-vacuum container that sets the wall temperature $T_{\text {wall }}$. As described earlier, the Penning and Paul traps differ in the mechanism to confine ions in the perpendicular plane, with the magnetic and $\mathrm{rf}$ fields preventing $\mathrm{mCP}$ propagation in the perpendicular direction for large enough $\epsilon$. On the other hand, for propagation along the axial direction, one can ignore these fields. However, in the axial direction, some other important effects need to be considered. In the order in which they occur, they are thermalization in walls, penetration of the double wall, the effect of the vacuum pump, and the static electric potential. In order to capture these effects, let us consider the following path for the mCPs:

(1) Outside at temperature $T_{\text {room }}$ with mCP density $n_{\text {lab }}$

(2) Metal $A$ with barrier $=\epsilon \phi_{1}$ at temperature $T_{\text {wall }}$ with density $n_{A}$ and volume $V_{A}$
(3) Buffer vacuum with effective temperature $T_{\text {wall }}$ with density $n_{\text {buf }}$ and volume $V_{\text {buf }}$

(4) Metal B with barrier $=\epsilon \phi_{2}$ at temperature $T_{\text {wall }}$ with density $n_{B}$ and volume $V_{\mathrm{B}}$

(5) Vacuum inside with density $n_{\text {vac }}$ and volume $V_{\text {vac }}$

In practice, there could be a series of metals at decreasing temperatures and varied work functions as described in Sec. II. We find that the smallest density inside the trap is obtained by two adjacent metals, both cold and decreasing work functions, and hence work with this simplified model, which nonetheless captures the inherent suppression. Note that the barrier for a positively charged particle to enter the metal from outside $\epsilon \phi$ is described in detail in Appendix 2.

The change in local number density in any segment is given by the sum of the fluxes along each wall in the axial direction, ignoring the perpendicular direction. For the metal $A$, the net incoming flux is

$$
\begin{aligned}
\text { flux }_{\text {in }}^{A}= & {\left[n_{\text {lab }} e^{-\left(\epsilon \phi_{1} / T_{\text {room }}\right)} \sqrt{\frac{T_{\text {room }}}{2 \pi m_{Q}}}\right.} \\
& \left.+n_{\text {buf }} e^{-\left(\epsilon \phi_{1} / T_{\text {wall }}\right)} \sqrt{\frac{T_{\text {wall }}}{2 \pi m_{Q}}}\right] .
\end{aligned}
$$

Here, the $\sqrt{T_{\text {room }} / 2 \pi m_{Q}}$ comes from the average velocity and the exponent from needing to penetrate the double layer. We can similarly write the outgoing flux, which gives

$$
\text { flux }_{\text {out }}^{A}=2 n_{A} \sqrt{\frac{T_{\text {wall }}}{2 \pi m_{Q}}} .
$$

Here, the exponent is absent because the double layer pushes out all the positive charges that reach the boundary and hence the flux is independent of the presence of the double layer. We can then write $\dot{n}=\left(A_{\text {in }} / V\right)$ flux $_{\text {in }}-$ $\left(A_{\text {out }} / V\right)$ flux $_{\text {out }}$ for the different parts:

$$
\begin{aligned}
\dot{n}_{A} & =\frac{A_{\text {wall }}}{V_{A}}\left[n_{\text {lab }} e^{-\left(\epsilon \phi_{1} / T_{\text {room }}\right)} \sqrt{\frac{T_{\text {room }}}{2 \pi m_{Q}}}+n_{\text {buf }} e^{-\left(\epsilon \phi_{1} / T_{\text {wall }}\right)} \sqrt{\frac{T_{\text {wall }}}{2 \pi m_{Q}}}-2 n_{A} \sqrt{\frac{T_{\text {wall }}}{2 \pi m_{Q}}}\right], \\
\dot{n}_{\text {buf }} & =\frac{A_{\text {wall }}}{V_{\text {buf }}} \sqrt{\frac{T_{\text {wall }}}{2 \pi m_{Q}}}\left[n_{A}+n_{B}-n_{\text {buf }}\left(e^{-\left(\epsilon \phi_{1} / T_{\text {wall }}\right)}+e^{-\left(\epsilon \phi_{2} / T_{\text {wall }}\right)}\right)\right], \\
\dot{n}_{B} & =\frac{A_{\text {wall }}}{V_{\text {ion }}} \sqrt{\frac{T_{\text {wall }}}{2 \pi m_{Q}}}\left(\left[n_{\text {buf }}+n_{\text {vac }}\right) e^{-\left(\epsilon \phi_{2} / T_{\text {wall }}\right)}-2 n_{B}\right], \\
\dot{n}_{\text {vac }} & =\sqrt{\frac{T_{\text {wall }}}{2 \pi m_{Q}}}\left[A_{\text {wall }} n_{B}-n_{\text {vac }}\left(A_{\text {wall }} e^{-\left(\epsilon \phi_{2} / T_{\text {wall }}\right)}+A_{\text {fan }}\right)\right] / V_{\text {vac }} .
\end{aligned}
$$


In the last line, the effect of the vacuum pump is taken into account by putting in an area $A_{\text {fan }}$ for the vacuum fan or other ion-evacuation mechanisms. The assumption here is that all mCPs that hit the area, $A_{\text {fan }}$, are effectively evacuated from the vacuum region. The steady-state solution to this is

$$
\begin{aligned}
n_{\text {vac }}^{\text {eqbm }} & =n_{\text {lab }} \frac{\sqrt{T_{\text {room }}}}{\sqrt{T_{\text {wall }}}} \frac{e^{-\left(\epsilon \phi_{1} / T_{\text {room }}\right)+\left(\epsilon \phi_{1} / T_{\text {wall }}\right)}}{\left[2\left(A_{\text {fan }} / A_{\text {wall }}\right) e^{\epsilon\left(\phi_{1}+\phi_{2}\right) / T_{\text {wall }}}+1\right]} \\
& \equiv n_{\text {on }}, \quad \text { fan on. }
\end{aligned}
$$

If the fan is turned off, the equilibrium density is set by $A_{\text {fan }} \rightarrow 0$ and given by

$$
n_{\text {off }}=n_{\text {lab }} \frac{\sqrt{T_{\text {room }}}}{\sqrt{T_{\text {wall }}}}\left[e^{\left(\epsilon \phi_{1} / T_{\text {wall }}\right)-\left(\epsilon \phi_{1} / T_{\text {room }}\right)}\right], \quad \text { fan off. }
$$

This contains an exponential enhancement in the limit $T_{\text {wall }} \ll T_{\text {room }}$. This happens because mCPs readily leak from the metal into the vacuum, subsequently get cooled to $T_{\text {wall }}$, and now have to overcome a barrier to reenter the metal. This exponential enhancement happens only with a hermetic metal container and is a potentially promising mechanism to greatly enhance the number density inside the trap. Since the hermeticity of the containers is unknown and in the spirit of being conservative, we never allow densities to put constraints on going above $n_{\text {lab }} \sqrt{T_{\text {room }} / T_{\text {wall }}}$.

Let us now discuss the dynamics after the fan is turned off. $n_{\text {vac }}$ is somewhere in between $n_{\text {off }}$ and $n_{\text {on }}$ The mCPs in metal $B$ diffuse into vacuum after the fan is turned off. Metal B has a number density that is relatively independent of the on or off status. Solving Eq. A3, it is given by

$$
n_{B}=\frac{\sqrt{T_{\text {room }}}}{\sqrt{T_{\text {wall }}}} \frac{n_{\text {lab }}}{2} e^{-\left(\epsilon \phi_{1} / T_{\text {room }}\right)} e^{-\left[\epsilon\left(\phi_{2}-\phi_{1}\right) / T_{\text {wall }}\right]} .
$$

The second term is an exponential enhancement. Assuming that the fan is turned off at time $\tau=0$, the number density in the vacuum after time $\tau$ is

$$
n_{\mathrm{vac}}(\tau)=n_{B} \frac{\tau}{L_{\mathrm{vac}}} \sqrt{\frac{T_{\mathrm{wall}}}{2 \pi m_{Q}}}+n_{\mathrm{on}},
$$

where $L_{\mathrm{vac}}=V_{\mathrm{vac}} / A_{\mathrm{vac}}$. This is solved in the small- $\tau$ limit: the maximum it can reach is $n_{\text {off. }}$ As noted earlier, $n_{\text {off }}$ contains an exponential enhancement that we cut off at $n_{\text {lab }} \sqrt{T_{\text {room }} / T_{\text {wall }}}$. Finally, then, the ambient number density in the trap vacuum is

$$
n_{\text {trap }}=\operatorname{Min}\left[n_{\mathrm{vac}}(\tau), n_{\mathrm{off}}, n_{\mathrm{lab}} \sqrt{\frac{T_{\text {room }}}{T_{\text {wall }}}}\right] \text {, }
$$

where $n_{\mathrm{vac}}(\tau)$ is the number density as the trap is filling with $\mathrm{mCPs}$ before it reaches equilibrium, as calculated in Eq. (A7).

The mCPs are thermalized to $T_{\text {wall }}$ inside the trap. Then, to find the number density at the position of the ion, we must take into account the axial potential barrier $V_{z}$. To do this, we take a Boltzmann factor on top of the average number density $n_{\text {trap }}$ inside the trap to finally find the number density at the position of the ion as follows:

$$
n_{\text {ion }}^{Q}=e^{-\left(\epsilon V_{z} / T_{\text {wall }}\right)} n_{\text {trap }} .
$$

Note that values of $V_{z}$ are listed in Table I. $n_{\text {ion }}^{Q}$ is the density that we use in the rate calculations.

\section{Work function of metals}

Here, we consider the effect of the work function of a metal on the passage of mCPs through that metal. This is only relevant for mCPs of relatively large charge $(\epsilon \gtrsim$ $10^{-2}$ ) but for those it can be a large effect. The work function for mCPs, which we call $\epsilon \phi$, is not simply $\epsilon$ times the work function for electrons. For electrons, the work function arises from several contributions (of varying sign) including, e.g., the binding to the lattice of nuclei, the Fermi sea of other electrons, and surface effects such as the "double layer" or the image-charge potential. Several of these do not apply to mCPs and so the final answer for the work function of an $\mathrm{mCP}$ in a metal is significantly different from $\epsilon$ times the work function for electrons.

The density of mCPs is low enough that any Fermi sea of other mCPs (if they are fermions) is not relevant. For electrons, removing the Fermi sea would increase the work function, making them more deeply bound to the metal than their normal work function.

The image charge of the $\mathrm{mCP}$ also has charge $\epsilon e$ and so the potential energy between the $\mathrm{mCP}$ and the image charge is $\propto \epsilon^{2}$ and is irrelevant for $\epsilon \lesssim 0.1$, which is the region that we consider. For electrons, the image-charge force is attractive and so removing it makes electrons less deeply bound compared to their normal work function.

Furthermore, we are considering positive $\mathrm{mCPs}$, so they do not bind to nuclei. Of course, they will have an electromagnetic interaction with the positively charged (pointlike) nuclei and the negatively charged (relatively uniform) sea of electrons. The only negative potential-energy contribution could come from binding with electrons but such a bound state would be much larger than the normal size of an atom (or interatomic spacing) by a factor $\epsilon^{-1}$. Within 
such a large distance, there will be many nuclei and electrons and thus a roughly zero net charge density. Note that the mCP itself is significantly heavier and thus of smaller wavelength than an electron in the entire parameter space that we consider. Therefore, its wave function is quite different than the wave function of the electron. The positive $\mathrm{mCP}$ is mainly repelled by the repulsive potentials of positively charged nuclei, so at most we would expect that the effect of interaction would be a repulsion of the $\mathrm{mCP}$, namely a positive potential energy relative to infinity. But this is unlikely to be very significant.

The "double-layer" surface effect is relevant for mCPs. Since the metal has negative charge density extending outside the region that contains the positive nuclei, it acts a bit like a capacitor around the edges of the metal. Namely, it has a potential-energy barrier for the mCPs that is simply $\epsilon$ times the magnitude of the usual double-layer contribution to the work function for electrons. We thus use this as our estimate for the work function (potential-energy barrier) for mCPs.

In Table II, we list the usual electron work functions (W) for several relevant metals surrounding the experiments that we consider. We also list the double-layer contribution (D) and the remainder of the work function $(\mu)$. Wherever available, this data is taken directly from Ref. [80] and the numbers for the rest of the metals are interpolated from the data available in Ref. [80] for different $r_{s}$. Note that we adopt conventions in which

$$
\mathrm{WF}=\mathrm{DL}-\mu .
$$

We then take the potential-energy barrier for the mCPs crossing a metal to be

$$
\epsilon \phi=\epsilon \times \mathrm{DL},
$$

where we adopt the convention that DL is positive.

As noted above in Appendix 1, the relevant effect of the work function of the metals around the experiment comes from the case where the experiment is encased in two different metals and the work function for mCPs of the inner metal is larger (in magnitude) then that of the outer one.

TABLE II. The electron work functions (WF) for several relevant metals surrounding the experiments that we consider. If not available in Ref. [80], interpolation is used to estimate the numbers using the known $r_{s}$ values. The double-layer contribution (DL) and the remainder of the work function $(\mu)$ are also shown.

\begin{tabular}{lcccc}
\hline \hline Element & $r_{s}$ & $\mu(\mathrm{eV})$ & $\mathrm{DL}(\mathrm{eV})$ & $\mathrm{WF}(\mathrm{eV})$ \\
\hline Copper & 2.67 & -0.45 & 3.19 & 3.65 \\
Steel & 2.12 & 2.05 & 5.91 & 3.86 \\
Aluminum & 2.07 & 2.39 & 6.26 & 3.87 \\
Gold & 3.01 & -1.20 & 2.30 & 3.5 \\
Nickel & 2.59 & -0.20 & 3.47 & 3.68 \\
\hline \hline
\end{tabular}

Note that for many of the experiments, the metals enclosing them are often grounded. This slightly modifies the above difference in work functions. If the two metals were not electrically connected, then we would just have the difference in the two mCP work functions, $\Delta \phi=\phi_{1}-\phi_{2}=$ $\mathrm{DL}_{1}-\mathrm{DL}_{2}$. But if they are connected by a wire (or both grounded), then one also subtracts from this difference in double layers the difference in electron WF, thus ending up with just the difference in the $\mu$ values. This is because of the contact potential effect between two metals. Thus, in this case, the potential barrier-height difference between the two metals is

$\Delta U=\epsilon\left[\left(\mathrm{DL}_{1}-\mathrm{DL}_{2}\right)-\left(\mathrm{WF}_{1}-\mathrm{WF}_{2}\right)\right]=\epsilon\left(\mu_{1}-\mu_{2}\right)$.

In this case, this would actually be what enters all the equations in Appendix 1, instead of the quantity $\epsilon\left(\phi_{1}-\phi_{2}\right)$. However, since the work functions of the relevant metals are not very different, this does not change our answer appreciably.

There are many paths that an $\mathrm{mCP}$ may take to enter each actual experiment, with multiple different metals to pass through. Furthermore, while the overall work functions of each metal are measured quantities, the part of the work function that comes from the double layer must be calculated and may have some uncertainty to it. Thus, in order to avoid all this complication, we simply take very conservative estimates for the $\mathrm{mCP}$ work functions. From Table II, it can be seen that the differences in the work functions that are relevant for any of the experiments is at most approximately $3 \mathrm{eV}$. Therefore, we assume that $\phi_{2}-\phi_{1}=3 \mathrm{eV}$ for all equations in Appendix 1. This sets the top of the excluded regions in Fig. 2 and on the right side in Fig. 3. Furthermore, we assume that $\phi_{1}=3 \mathrm{eV}$ (and so $\phi_{2}=6 \mathrm{eV}$ ) wherever relevant, which is conservative.

\section{APPENDIX B: HEATING RATES}

Consider mCPs $m_{Q}$ scattering with an ion target. Let us assume that their velocities are $\mathbf{v}_{\mathbf{Q}}$ and $\mathbf{v}_{\text {ion }}$. The c.m. velocity of each particle is given by

$$
\mathbf{v}_{\text {ion }}{ }^{\text {c.m. }, i}=\left(\mathbf{v}_{\text {ion }}-\mathbf{v}_{\mathbf{Q}}\right) \frac{m_{Q}}{m_{\text {ion }}+m_{Q}}
$$

and the final velocity is

$$
\mathbf{v}_{\text {ion }}{ }^{\text {c.m. } . f}=v_{\text {ion }}^{\text {c.m. } i} \hat{n} .
$$

Here, we use the fact that the magnitude of the velocity does not change in the c.m. frame. The final velocity of the ion is assumed to be in the $\hat{\mathbf{n}}$ direction. 
The change in velocity in any frame is

$$
\begin{aligned}
\Delta \mathbf{v}_{\text {ion }}= & \frac{m_{Q}}{m_{\text {ion }}+m_{Q}}\left|\mathbf{v}_{\text {ion }}-\mathbf{v}_{\mathbf{Q}}\right|\left(\hat{\mathbf{n}}-\frac{\mathbf{v}_{\text {ion }}-\mathbf{v}_{\mathbf{Q}}}{\left|v_{\text {ion }}-v_{Q}\right|}\right) \\
= & \frac{m_{Q}}{m_{\text {ion }}+m_{Q}}\left[(\cos \theta-1)\left(\mathbf{v}_{\text {ion }}-\mathbf{v}_{\mathbf{Q}}\right)\right. \\
& \left.+\sin \theta\left|\mathbf{v}_{\text {ion }}-\mathbf{v}_{\mathbf{Q}}\right| \mathbf{n}_{\perp}\right] .
\end{aligned}
$$

We can drop the $\sin \theta$ term because it averages to zero. The

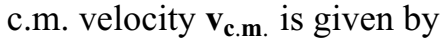

$$
\mathbf{v}_{\mathbf{c} . \mathbf{m} .}=\frac{\left(m_{\mathrm{ion}} \mathbf{v}_{\text {ion }}+m_{Q} \mathbf{v}_{\mathbf{Q}}\right)}{m_{\text {ion }}+m_{Q}}
$$

The energy transfer is given by

$$
\Delta E_{\text {ion }}=m_{\text {ion }} \mathbf{v}_{\mathbf{c} . \mathbf{m}} \cdot \Delta \mathbf{v}_{\text {ion }}
$$

We simplify this equation in the simple case of free-ion targets, in order to learn qualitative features. In the free-ion limit, there is no minimum energy transfer $E_{\min }$. Hence, the energy transfer rate is given by

$$
\begin{aligned}
\dot{H}= & n_{Q}\left\langle\sigma \Delta E_{\text {ion }} v_{\text {rel }}\right\rangle \\
= & \frac{n_{Q} m_{Q} m_{\text {ion }}}{m_{\text {ion }}+m_{Q}} \int d^{3} v_{Q} g_{Q} \int d^{3} v_{\text {ion }} g_{\text {ion }} \int d \cos \theta \\
& \times \frac{d \sigma}{d \cos \theta}(1-\cos \theta)\left|\mathbf{v}_{\mathbf{Q}}-\mathbf{v}_{\text {ion }}\right| \mathbf{v}_{\mathbf{c} . \mathbf{m} .} \cdot\left(\mathbf{v}_{\mathbf{Q}}-\mathbf{v}_{\text {ion }}\right) \\
= & \frac{n_{Q} m_{Q} m_{\text {ion }}}{m_{\text {ion }}+m_{Q}} \int d^{3} v_{Q} g_{Q} \int d^{3} v_{\text {ion }} g_{\text {ion }}\left|\mathbf{v}_{\mathbf{Q}}-\mathbf{v}_{\text {ion }}\right| \mathbf{v}_{\mathbf{c} . \mathbf{m} .} \\
& \cdot\left(\mathbf{v}_{\mathbf{Q}}-\mathbf{v}_{\text {ion }}\right) \sigma_{\text {ion }}\left(\left|\mathbf{v}_{\mathbf{Q}}-\mathbf{v}_{\text {ion }}\right|\right) .
\end{aligned}
$$

Here, $\sigma_{\text {ion }}$ is the transfer cross section for Rutherford scattering.

We now do a change of variable:

$$
\begin{aligned}
& \mathbf{v}_{\text {rel }}=\mathbf{v}_{\mathbf{Q}}-\mathbf{v}_{\text {ion }} \\
& \mathbf{v}_{\mathbf{m}}=\frac{\left(m_{Q} / T_{Q}\right) \mathbf{v}_{\mathbf{Q}}+\left(m_{\text {ion }} / T_{\text {ion }}\right) \mathbf{v}_{\text {ion }}}{\left(m_{Q} / T_{Q}\right)+\left(m_{\text {ion }} / T_{\text {ion }}\right)} .
\end{aligned}
$$

Then,

$$
\int d^{3} v_{Q} g_{Q} \int d^{3} v_{\text {ion }} g_{\text {ion }}=\int d^{3} v_{\text {rel }} f_{\text {rel }} \int d^{3} v_{m} f_{m}
$$

The thermal width of $f_{\text {rel }}$ is given by $\left(T_{Q} / m_{Q}\right)+$ $\left(T_{\text {ion }} / m_{\text {ion }}\right)$. Also,

$$
\mathbf{v}_{\mathbf{c} . \mathbf{m} .}=\mathbf{v}_{\mathbf{m}}+\frac{m_{\mathrm{ion}} m_{Q}\left(T_{Q}-T_{\mathrm{ion}}\right)}{\left(m_{\mathrm{ion}}+m_{Q}\right)\left(m_{Q} T_{\mathrm{ion}}+m_{\mathrm{ion}} T_{Q}\right)} \mathbf{v}_{\mathrm{rel}} .
$$

Then,

$$
\begin{aligned}
\dot{H}= & \frac{n_{Q} m_{Q} m_{\text {ion }}}{m_{\text {ion }}+m_{Q}} \int d^{3} v_{\text {rel }} f_{\text {rel }} \int d^{3} v_{m} f_{m}\left|\mathbf{v}_{\text {rel }}\right| \\
& \times\left[\mathbf{v}_{\mathbf{m}}+\frac{m_{\text {ion }} m_{Q}\left(T_{Q}-T_{\text {ion }}\right)}{\left(m_{\text {ion }}+m_{Q}\right)\left(m_{Q} T_{\text {ion }}+m_{\text {ion }} T_{Q}\right)} \mathbf{v}_{\text {rel }}\right] \\
& \times \mathbf{v}_{\text {rel }} \sigma_{\text {ion }}\left(\left|\mathbf{v}_{\text {rel }}\right|\right) .
\end{aligned}
$$

Now, the term proportional to just $\mathbf{v}_{\mathbf{m}}$ is odd and hence its integral is zero. The rest is independent of $v_{m}$ and hence the Boltzmann integral $v_{m}$ gives 1 . This yields

$$
\begin{aligned}
\dot{H}= & \frac{n_{Q} m_{\text {ion }}^{2} m_{Q}^{2}\left(T_{Q}-T_{\text {ion }}\right)}{\left(m_{\text {ion }}+m_{Q}\right)^{2}\left(m_{Q} T_{\text {ion }}+m_{\text {ion }} T_{Q}\right)} \\
& \times \int d^{3} v_{\text {rel }} f_{\text {rel }} v_{\text {rel }}^{3} \sigma_{t}\left(v_{\text {rel }}\right) .
\end{aligned}
$$

When $\sigma_{t}=\sigma_{0} / v_{\text {rel }}^{4}$, then

$$
\int d^{3} v_{\text {rel }} \frac{f_{\text {rel }}}{v_{\text {rel }}}=\left[\frac{2}{\pi} \frac{m_{\text {ion }} m_{Q}}{\left(m_{Q} T_{\text {ion }}+m_{\text {ion }} T_{Q}\right)}\right]^{1 / 2} .
$$

If we define $\left(T_{\text {ion }} / m_{\text {ion }}\right)+\left(T_{Q} / m_{Q}\right)=u_{\text {th }}^{2}$, we obtain

$$
\dot{H}=\sqrt{\frac{2}{\pi}} \frac{n_{Q} m_{Q} m_{\text {ion }}\left(T_{Q}-T_{\text {ion }}\right)}{\left(m_{\text {ion }}+m_{Q}\right)^{2}} \frac{\sigma_{0}}{u_{\mathrm{th}}^{3}} .
$$

We see that the heating rate is proportional to the difference in temperatures $T_{Q}-T_{\text {ion. }}$. Furthermore, the rate is also proportional to $u_{\mathrm{th}}^{-3}$.

However, the ions are in a harmonic oscillator potential and hence there is a minimum energy $E_{\min }$ such that

$$
\Delta E_{\text {ion }}>E_{\text {min }}
$$

In this case, the heating rate is instead

$$
\begin{aligned}
\dot{H}= & n_{\text {ion }}^{Q} \int d^{3} \mathbf{v}_{\mathbf{Q}} g_{Q} \int d^{3} \mathbf{v}_{\text {ion }} g_{\text {ion }} \int d \Omega\left|v_{Q}-v_{\text {ion }}\right| \\
& \times \frac{d \sigma}{d \Omega} E_{\text {ion }} \Theta\left(\left|E_{\text {ion }}\right|-E_{\text {thr }}\right) \Theta\left(E_{\text {samp }}-\left|E_{\text {ion }}\right|\right) .
\end{aligned}
$$

[1] E. Izaguirre and I. Yavin, New window to millicharged particles at the LHC, Phys. Rev. D 92, 035014 (2015).

[2] R. Akers, G. Alexander, J. Allison, K. Ametewee, K. Anderson, S. Arcelli, S. Asai, D. Axen, G. Azuelos, A. Ball, et al., Search for heavy charged particles and for particles with anomalous charge in $e^{+} e^{-}$collisions at LEP, Z. Phys. C Part. Fields 67, 203 (1995). 
[3] A. A. Prinz, R. Baggs, J. Ballam, S. Ecklund, C. Fertig, J. A. Jaros, K. Kase, A. Kulikov, W. G. J. Langeveld, R. Leonard, T. Marvin, T. Nakashima, W. R. Nelson, A. Odian, M. Pertsova, G. Putallaz, and A. Weinstein, Search for Millicharged Particles at SLAC, Phys. Rev. Lett. 81, 1175 (1998).

[4] G. Magill, R. Plestid, M. Pospelov, and Y.-D. Tsai, Millicharged Particles in Neutrino Experiments, Phys. Rev. Lett. 122, 071801 (2019).

[5] R. Acciarri, et al., (Collaboration ArgoNeuT), Improved Limits on Millicharged Particles Using the ArgoNeuT Experiment at Fermilab, Phys. Rev. Lett. 124, 131801 (2020).

[6] A. Ball, et al., Search for millicharged particles in protonproton collisions at $\sqrt{s}=13 \mathrm{TeV}$, Phys. Rev. D 102, 032002 (2020).

[7] G. Marocco and S. Sarkar, Blast from the past: Constraints on the dark sector from the BEBC WA66 beam dump experiment, SciPost Phys. 10, 043 (2021).

[8] A. Ball et al., A letter of intent to install a milli-charged particle detector at LHC P5, arXiv:1607.04669 (2016).

[9] A. Berlin, N. Blinov, G. Krnjaic, P. Schuster, and N. Toro, Dark matter, millicharges, axion and scalar particles, gauge bosons, and other new physics with LDMX, Phys. Rev. D 99, 075001 (2019).

[10] K. J. Kelly and Y.-D. Tsai, Proton fixed-target scintillation experiment to search for millicharged dark matter, Phys. Rev. D 100, 015043 (2019).

[11] R. Harnik, Z. Liu, and O. Palamara, Millicharged particles in liquid argon neutrino experiments, JHEP 2019, 170 (2019).

[12] S. Davidson, S. Hannestad, and G. Raffelt, Updated bounds on millicharged particles, JHEP 2000, 003 (2000).

[13] J. H. Chang, R. Essig, and S. D. McDermott, Supernova 1987A constraints on sub-GeV dark sectors, millicharged particles, the QCD axion, and an axion-like particle, JHEP 2018, 051 (2018).

[14] C. Dvorkin, T. Lin, and K. Schutz, Making dark matter out of light: Freeze-in from plasma effects, Phys. Rev. D 99, 115009 (2019).

[15] C. Creque-Sarbinowski, L. Ji, E. D. Kovetz, and M. Kamionkowski, Direct millicharged dark matter cannot explain the EDGES signal, Phys. Rev. D 100, 023528 (2019).

[16] M. Ambrosio, et al., (Collaboration MACRO), Final search for lightly ionizing particles with the MACRO detector, arXiv:hep-ex/0402006 (2004).

[17] R. Agnese, et al., (Collaboration CDMS), First Direct Limits on Lightly Ionizing Particles with Electric Charge Less Than e/6, Phys. Rev. Lett. 114, 111302 (2015).

[18] S. Knapen, T. Lin, M. Pyle, and K. M. Zurek, Detection of light dark matter with optical phonons in polar materials, Phys. Lett. B 785, 386 (2018).

[19] S. Alvis, I. Arnquist, F. Avignone III, A. Barabash, C. Barton, F. Bertrand, V. Brudanin, M. Busch, M. Buuck, T. Caldwell, et al., First Limit on the Direct Detection of Lightly Ionizing Particles for Electric Charge as Low as $e / 1000$ with the Majorana Demonstrator, Phys. Rev. Lett. 120, 211804 (2018).

[20] L. Singh, et al., (Collaboration TEXONO), Constraints on millicharged particles with low threshold germanium detectors at Kuo-Sheng reactor neutrino laboratory, Phys. Rev. D 99, 032009 (2019).

[21] C. Blanco, J. I. Collar, Y. Kahn, and B. Lillard, Dark matterelectron scattering from aromatic organic targets, Phys. Rev. D 101, 056001 (2020).

[22] R. Essig, J. Pérez-Ríos, H. Ramani, and O. Slone, Direct detection of spin-(in)dependent nuclear scattering of sub$\mathrm{GeV}$ dark matter using molecular excitations, Phys. Rev. Res. 1, 033105 (2019).

[23] A. Berlin, R. T. D’Agnolo, S. A. R. Ellis, P. Schuster, and N. Toro, Directly Deflecting Particle Dark Matter, Phys. Rev. Lett. 124, 011801 (2020).

[24] N. A. Kurinsky, T. C. Yu, Y. Hochberg, and B. Cabrera, Diamond detectors for direct detection of sub-GeV dark matter, Phys. Rev. D 99, 123005 (2019).

[25] I. Alkhatib, et al., (Collaboration SuperCDMS), Constraints on Lightly Ionizing Particles from CDMSlite, Phys. Rev. Lett. 127, 081802 (2021).

[26] L. Barak, et al., (Collaboration SENSEI), SENSEI: DirectDetection Results on sub-GeV Dark Matter from a New Skipper-CCD, Phys. Rev. Lett. 125, 171802 (2020).

[27] S. M. Griffin, Y. Hochberg, K. Inzani, N. Kurinsky, T. Lin, and T. Chin, Silicon carbide detectors for sub-GeV dark matter, Phys. Rev. D 103, 075002 (2021).

[28] R. Barkana, N. J. Outmezguine, D. Redigolo, and T. Volansky, Strong constraints on light dark matter interpretation of the EDGES signal, Phys. Rev. D 98, 103005 (2018).

[29] J. B. Muñoz and A. Loeb, A small amount of mini-charged dark matter could cool the baryons in the early universe, Nature 557, 684 (2018).

[30] H. Liu, N. J. Outmezguine, D. Redigolo, and T. Volansky, Reviving millicharged dark matter for 21-cm cosmology, Phys. Rev. D 100, 123011 (2019).

[31] N. Kurinsky, D. Baxter, Y. Kahn, and G. Krnjaic, Dark matter interpretation of excesses in multiple direct detection experiments, Phys. Rev. D 102, 015017 (2020).

[32] I. M. Bloch, A. Caputo, R. Essig, D. Redigolo, M. Sholapurkar, and T. Volansky, Exploring new physics with $\mathcal{O}(\mathrm{keV})$ electron recoils in direct detection experiments, JHEP 2021, 178 (2021).

[33] R. Harnik, R. Plestid, M. Pospelov, and H. Ramani, Millicharged cosmic rays and low recoil detectors, Phys. Rev. D 103, 075029 (2021).

[34] T. Emken, R. Essig, C. Kouvaris, and M. Sholapurkar, Direct detection of strongly interacting sub-GeV dark matter via electron recoils, JCAP 2019, 070 (2019).

[35] M. Pospelov and H. Ramani, Earth-bound millicharge relics, Phys. Rev. D 103, 115031 (2021).

[36] P. C. Kim, E. R. Lee, I. T. Lee, M. L. Perl, V. Halyo, and D. Loomba, Search for Fractional-Charge Particles in Meteoritic Material, Phys. Rev. Lett. 99, 161804 (2007).

[37] D. C. Moore, A. D. Rider, and G. Gratta, Search for Millicharged Particles Using Optically Levitated Microspheres, Phys. Rev. Lett. 113, 251801 (2014).

[38] G. Afek, F. Monteiro, J. Wang, B. Siegel, S. Ghosh, and D. C. Moore, Limits on the abundance of millicharged particles bound to matter, Preprint ArXiv:2012.08169 (2020).

[39] D. Hanneke, S. F. Hoogerheide, and G. Gabrielse, Cavity control of a single-electron quantum cyclotron: Measuring the electron magnetic moment, Phys. Rev. A 83, 052122 (2011). 
[40] W. B. Cairncross, D. N. Gresh, M. Grau, K. C. Cossel, T. S. Roussy, Y. Ni, Y. Zhou, J. Ye, and E. A. Cornell, Precision Measurement of the Electron's Electric Dipole Moment Using Trapped Molecular Ions, Phys. Rev. Lett. 119, 153001 (2017).

[41] G. Schneider, A. Mooser, M. Bohman, N. Schön, J. Harrington, T. Higuchi, H. Nagahama, S. Sellner, C. Smorra, $\mathrm{K}$. Blaum, et al., Double-trap measurement of the proton magnetic moment at 0.3 parts per billion precision, Science 358, 1081 (2017).

[42] C. Smorra, S. Sellner, M. Borchert, J. Harrington, T. Higuchi, H. Nagahama, T. Tanaka, A. Mooser, G. Schneider, M. Bohman, et al., A parts-per-billion measurement of the antiproton magnetic moment, Nature 550, 371 (2017).

[43] B. M. Roberts, et al., Search for transient variations of the fine structure constant and dark matter using fiber-linked optical atomic clocks, New J. Phys. 22, 093010 (2020).

[44] C. Smorra, Y. Stadnik, P. Blessing, M. Bohman, M. Borchert, J. Devlin, S. Erlewein, J. Harrington, T. Higuchi, A. Mooser, et al., Direct limits on the interaction of antiprotons with axion-like dark matter, Nature 575, 310 (2019).

[45] H. Häffner, C. F. Roos, and R. Blatt, Quantum computing with trapped ions, Phys. Rep. 469, 155 (2008).

[46] D. A. Hite, Y. Colombe, A. C. Wilson, K. R. Brown, U. Warring, R. Jördens, J. D. Jost, K. McKay, D. Pappas, D. Leibfried, et al., 100-Fold Reduction of Electric-Field Noise in an Ion Trap Cleaned with in situ Argon-Ion-Beam Bombardment, Phys. Rev. Lett. 109, 103001 (2012).

[47] D. Carney, H. Häffner, D. C. Moore, and J. M. Taylor, Trapped Electrons and Ions as Particle Detectors, Phys. Rev. Lett. 127, 061804 (2021).

[48] M. Safronova, D. Budker, D. DeMille, D. F. J. Kimball, A. Derevianko, and C. W. Clark, Search for new physics with atoms and molecules, Rev. Mod. Phys. 90, 025008 (2018).

[49] S. Sellner, M. Besirli, M. Bohman, M. Borchert, J. Harrington, T. Higuchi, A. Mooser, H. Nagahama, G. Schneider, C. Smorra, et al., Improved limit on the directly measured antiproton lifetime, New J. Phys. 19, 083023 (2017).

[50] J. F. Goodwin, G. Stutter, R. C. Thompson, and D. M. Segal, Resolved-Sideband Laser Cooling in a Penning Trap, Phys. Rev. Lett. 116, 143002 (2016).

[51] S. Mavadia, G. Stutter, J. Goodwin, D. Crick, R. Thompson, and D. Segal, Optical sideband spectroscopy of a single ion in a Penning trap, Phys. Rev. A 89, 032502 (2014).

[52] M. Borchert, P. Blessing, J. Devlin, J. Harrington, T. Higuchi, J. Morgner, C. Smorra, E. Wursten, M. Bohman, M. Wiesinger, et al., Measurement of Ultralow Heating Rates of a Single Antiproton in a Cryogenic Penning Trap, Phys. Rev. Lett. 122, 043201 (2019).

[53] G. Schneider, Ph.D. thesis, Johannes Gutenberg-Universität, Mainz, 2017.

[54] A. Mooser, A. Rischka, A. Schneider, K. Blaum, S. Ulmer, and J. Walz, in Journal of Physics: Conference Series (IOP Publishing, 2018), Vol. 1138, p. 012004.

[55] S. Ulmer, C. C. Rodegheri, K. Blaum, H. Kracke, A. Mooser, W. Quint, and J. Walz, Observation of Spin Flips with a Single Trapped Proton, Phys. Rev. Lett. 106, 253001 (2011).

[56] T. Savard, K. O'hara, and J. Thomas, Laser-noise-induced heating in far-off resonance optical traps, Phys. Rev. A 56, R1095 (1997).
[57] A. Mooser, H. Kracke, K. Blaum, S. A. Bräuninger, K. Franke, C. Leiteritz, W. Quint, C. C. Rodegheri, S. Ulmer, and J. Walz, Resolution of Single Spin Flips of a Single Proton, Phys. Rev. Lett. 110, 140405 (2013).

[58] M. Brownnutt, M. Kumph, P. Rabl, and R. Blatt, Ion-trap measurements of electric-field noise near surfaces, Rev. Mod. Phys. 87, 1419 (2015).

[59] Q. A. Turchette, B. King, D. Leibfried, D. Meekhof, C. Myatt, M. Rowe, C. Sackett, C. Wood, W. Itano, C. Monroe, et al., Heating of trapped ions from the quantum ground state, Phys. Rev. A 61, 063418 (2000).

[60] N. Daniilidis, S. Narayanan, S. A. Möller, R. Clark, T. E. Lee, P. J. Leek, A. Wallraff, S. Schulz, F. Schmidt-Kaler, and H. Häffner, Fabrication and heating rate study of microscopic surface electrode ion traps, New J. Phys. 13, 013032 (2011).

[61] G. Poulsen, Y. Miroshnychenko, and M. Drewsen, Efficient ground-state cooling of an ion in a large room-temperature linear Paul trap with a sub-hertz heating rate, Phys. Rev. A 86, 051402 (2012).

[62] We leave to upcoming work the calculation of the accumulation of millicharged dark matter in cases where the electric and magnetic fields are relevant [81]. Once such calculations are complete, our given limits on the number density in the laboratory can be translated to limits on the dark matter fraction in these cases.

[63] Millicharged particles with masses below $1 \mathrm{GeV}$ can accumulate on Earth temporarily before evaporating [35]. We leave limits on these masses for future work.

[64] D. A. Neufeld, G. R. Farrar, and C. F. McKee, Dark matter that interacts with baryons: Density distribution within the earth and new constraints on the interaction cross-section, Astrophys. J. 866, 111 (2018).

[65] The sensitivity is limited to be below $10^{-3}$ charge because of the large trap potential, as discussed in Sec. V.

[66] The final answer is only logarithmically sensitive to this time scale.

[67] In principle, energy transfers $E_{\text {ion }}=n \times \omega$, where all integers $n \geq 1$ are allowed. We restrict to $E_{\text {ion }} \geq 10 \times \omega$, a conservative choice that helps avoid form-factor calculations.

[68] See, e.g., Ref. [82] from the same group, where the potential depth is reported to be $V_{z}=5 \mathrm{mV}$.

[69] P. Micke, T. Leopold, S. King, E. Benkler, L. Spieß, L. Schmoeger, M. Schwarz, J. C. López-Urrutia, and P. Schmidt, Coherent laser spectroscopy of highly charged ions using quantum logic, Nature 578, 60 (2020).

[70] K. A. Gilmore, M. Affolter, R. J. Lewis-Swan, D. Barberena, E. Jordan, A. M. Rey, and J. J. Bollinger, Quantumenhanced sensing of displacements and electric fields with two-dimensional trapped-ion crystals, Science 373, 673 (2021).

[71] C. Matthiesen, Q. Yu, J. Guo, A. M. Alonso, and H. Häffner, Trapping Electrons in a Room-Temperature Microwave Paul Trap, Phys. Rev. X 11, 011019 (2021).

[72] Note that ion traps significantly suppress the black-body background because the ion is heavier than the electron and so has a lower scattering cross section with photons, and the trap frequencies are generally lower, so there are fewer photons in the black-body spectrum at those lower frequencies and those frequencies are below the lowest cutoff of the effective electromagnetic cavity surrounding the ion. 
[73] J. Cao, P. Zhang, J. Shang, K. Cui, J. Yuan, S. Chao, S. Wang, H. Shu, and X. Huang, A transportable ${ }^{4} \mathrm{OCa}+$ single-ion clock with $7.7 \times 10^{-17}$ systematic uncertainty, Preprint ArXiv:1607.03731 (2016).

[74] M. Delehaye and C. Lacroûte, Single-ion, transportable optical atomic clocks, J. Mod. Opt. 65, 622 (2018).

[75] M. Gellesch, J. Jones, R. Barron, A. Singh, Q. Sun, K. Bongs, and Y. Singh, Transportable optical atomic clocks for use in out-of-the-lab environments, Adv. Opt. Technol. 9, 313 (2020).

[76] J. R. C. López-Urrutia, A. Dorn, R. Moshammer, and J. Ullrich, The Freiburg electron beam ion trap/source project FreEBIT, Phys. Scr. T80, 502 (1999).

[77] A. Megía-Macías, R. Gebel, and B. Lefort, The ion source for the commissioning of ELENA ring, AIP Conf. Proc. 2011, 090014 (2018).
[78] M. Steck and Y. A. Litvinov, Heavy-ion storage rings and their use in precision experiments with highly charged ions, Prog. Part. Nucl. Phys. 115, 103811 (2020).

[79] R. von Hahn, A. Becker, F. Berg, K. Blaum, C. Breitenfeldt, H. Fadil, F. Fellenberger, M. Froese, S. George, J. Göck, et al., The cryogenic storage ring CSR, Rev. Sci. Instrum. 87, 063115 (2016).

[80] N. Lang and W. Kohn, Theory of metal surfaces: Work function, Phys. Rev. B 3, 1215 (1971).

[81] A. Berlin, H. Liu, M. Pospelov, and H. Ramani, Terrestrial millicharge densities, Upcoming.

[82] U. Warring, C. Ospelkaus, Y. Colombe, K. R. Brown, J. Amini, M. Carsjens, D. Leibfried, and D. J. Wineland, Techniques for microwave near-field quantum control of trapped ions, Phys. Rev. A 87, 013437 (2013). 